\title{
Symmetric Boundary Condition for Laplacian on Net of Regular Hexagons
}

\author{
Daniel Lee ${ }^{1}$ \\ ${ }^{1}$ Department of Applied Mathematics, Tunghai University, Taichung, Taiwan R.O.C. \\ Correspondence: Daniel Lee, Department of Applied Mathematics, Tunghai University, Taichung, Taiwan R.O.C. \\ E-mail: danlee@ thu.edu.tw
}

Received: April 8, 2017 Accepted: May 18, 2017 Online Published: May 25, 2017

doi:10.5539/jmr.v9n3p46 URL: https://doi.org/10.5539/jmr.v9n3p46

\begin{abstract}
Hexagonal grid methods are found useful in many research works, including numerical modeling in spherical coordinates, in atmospheric and ocean models, and simulation of electrical wave phenomena in cardiac tissues. Almost all of these used standard Laplacian and mostly on one configuration of regular hexagons. In this work, discrete symmetric boundary condition and energy product for anisotropic Laplacian are investigated firstly on general net of regular hexagons, and then generalized to its most extent in two- or three-dimensional cell-center finite difference applications up to the usage of symmetric stencil in central differences. For analysis of Laplacian related applications, this provides with an approach in addition to the M-matrix theory, series method, functional interpolations and Fourier vectors.
\end{abstract}

Keywords: Bilinear form, Cell-center finite difference, Discrete Laplacian, Hexagonal grid method.

\section{Introduction}

Hexagonal (Hex) grid methods are of interest in many research studies: (Pickering,1986) on direct method, (Makarov, Mararov \& Moskal'kov, 1993) giving a formula without proof, (Bystrytskyi \& Mosklkov, 2001) on seven-point method on rectangular grid with explicit form of eigenpairs, (Zhou \& Fulton, 2009) with periodic boundary condition (BC), (Heikes \& Randall, 1995, part I,II) and (Heikes, Randall \& Konor, 2013) on numerical modeling in spherical coordinates, (van Eck $\&$ Kors, 2005) on action potential in heart modeling via algebraic method without using diffusion in form of differential equation, (Nickovic,Gavrilov \& Tosic, 2002) showing advantages of Hex grids over commonly used square grids for use in atmospheric and ocean models. In the article by (Lee,Tien,Luo and Luk,2014), Hex grid finite difference (FD) methods are derived in a finite volume (FV) approach involving standard Laplacian, and used in the simulation of electrical wave phenomena propagated in two-dimensional reversed-C type cardiac tissues, exhibiting both linear and spiral waves more efficiently than similar computation carried on rectangular FVs. We note these cited works all used standard Laplacian and mostly on one configuration of regular hexagons.

In two-dimensional applications of configurations consisting of (subset of) Cartesian type regular hexagons, we denote the radius of hexagons by $r$, the height by $h\left(=\frac{\sqrt{3}}{2} r\right)$, and the center-to-center distance by $d(=2 h)$. Near a typical center node, $P_{0}=\left(x_{0}, y_{0}\right)$, the six neighbor (center) nodes are

$$
P_{j}=\left(x_{j}, y_{j}\right)=\left(x_{0}, y_{0}\right)+d\left(\cos \xi_{j}, \sin \xi_{j}\right), \quad \xi_{j}=\varphi+\frac{j \pi}{3}+\frac{\pi}{6}, \quad 1 \leq j \leq 6 .
$$

Here the phase angle, $\varphi$, is the configurarion parameter. Two particular instances are called type I $(\varphi=0)$ and type II $\left(\varphi=\frac{-\pi}{6}\right)$ for convenience. Hexagon centers in lattices of these two types are indexed as for an orthogonal Cartesian mesh as shown in Table 1, while the geometry and neighborhood of a general Hex FV shown in Table 2. Indexing rules are illustrated in Figs. 1, 2, 3 and 4.

For convenience, we abuse the notations and denote FV centers in a neighborhood (Figs. 3 and 4) by an ordered list,

$$
\begin{aligned}
\text { Type I : }\left\{P_{j}\right\}_{j=0}^{6}=\left\{P, P_{N}, P_{N W}, P_{S W}, P_{S}, P_{S E}, P_{N E}\right\}, \\
\text { Type II : }\left\{P_{j}\right\}_{j=0}^{6}=\left\{P, P_{N E}, P_{N W}, P_{W}, P_{S W}, P_{N W}, P_{E}\right\} .
\end{aligned}
$$

We note for applications that a two-dimensional irregular domain may be approximated by a sequence of (not necessarily Cartesian) nets of hexagons. Actually, our work in numerical modeling of ECG depends on this (Algorithm 1 in (Lee,Tien,Luo \& Luk, 2014)). 


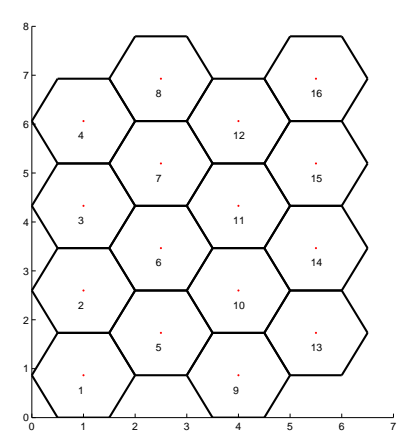

Figure 1. Lattice of type I regular hexagons in natural order by columns

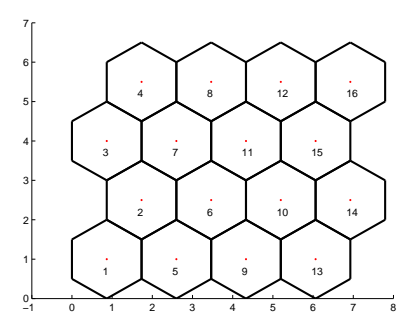

Figure 2. Lattice of type II regular hexagons in natural order by columns

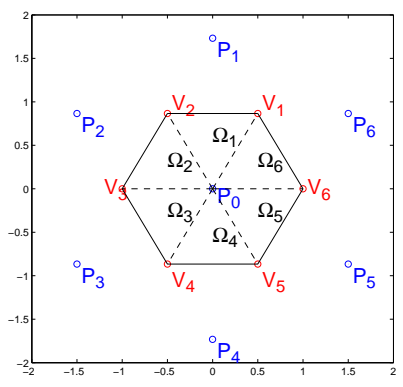

Figure 3. Type I regular hexagonal FV neighborhood

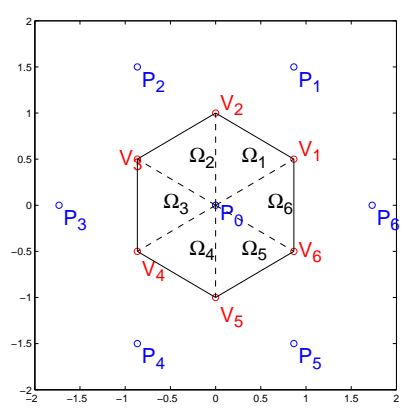

Figure 4. Type II regular hexagonal FV neighborhood 
Table 1. Lattices of type I and II regular hexagons

\begin{tabular}{cccc}
\hline Phase angle & \multicolumn{2}{c}{ Type I, $\varphi=0$} & \multicolumn{2}{c}{ Type II, $\varphi=-\pi / 6$} \\
\hline Center point & \multicolumn{2}{c}{$i^{\text {even }} i^{\text {odd }}$} & \multicolumn{2}{c}{$j^{\text {even }} j^{\text {odd }}$} \\
$c x(i, j)$ & $(1.5 i-0.5) r$ & $2 i h \quad(2 i-1) h$ \\
$c y(i, j)$ & $2 j h \quad(2 j-1) h$ & $(1.5 j-0.5) r$ \\
\hline
\end{tabular}

Table 2. Local geometry at a regular hexagon : six vertices and six neighbor centers

\begin{tabular}{cc}
\hline Phase angle & $\varphi \in \mathbb{R}$ \\
\hline Vertices & $V_{k}=(v x(*, k), v y(*, k)), \quad k=1,2, \cdots, 6$. \\
$v x(*, k)$ & $c x(*)+r \cos \left(\varphi+\frac{k \pi}{3}\right)$ \\
$v y(*, k)$ & $c y(*)+r \sin \left(\varphi+\frac{k \pi}{3}\right)$ \\
Neighbor centers & $P_{k}=V_{k}+V_{k+1}-P_{0}, \quad k=1,2, \cdots, 6$. \\
\hline
\end{tabular}

Concerning the negative anisotropic Laplacian

$$
-\operatorname{Lap}(f):=-D_{1} f_{x x}-D_{2} f_{y y}
$$

with positive constant diffusivities $D_{1}$ and $D_{2}$, we observe the following.

Lemma 1 (Reflection principle for anisotropic Laplacian.) The two configurations, type I and II regular hexagons centered at the origin together with the anisotropic Laplacian, are convertible from each other by applying reflection with respect to the main diagonal in the $x y$-plane, and therefore interchanging the two symbol lists (Figs. 3 and 4)

$$
\left\{x, y, D_{1}, D_{2}, N, N W, S W, S, S E, N E\right\} \quad \text { and } \quad\left\{y, x, D_{2}, D_{1}, E, S E, S W, W, N W, N E\right\} .
$$

With a general phase angle, the reflection interchanges

$$
\left\{(\varphi),\left(P_{j}\right)_{j=1}^{6}\right\} \quad \text { and } \quad\left\{\left(\frac{\pi}{2}-\varphi\right),\left(P_{6-j \% 6}\right)_{j=1}^{6}\right\} .
$$

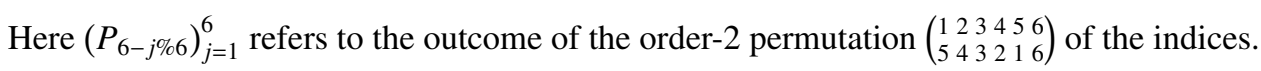

The focus in subsequent discussion is on net of type I hexagons.

We note that spectral analysis of iterative methods solving the discrete anisotropic Laplacian on a net of hexagons seems not as easy as the analysis on square grids (Suli,1993) and (Karaa \& Zhang, 2003), since finite trigonometric series is incomplete for the error analysis (even) on a single regular hexagon (McCartin,2002,2003).

The analysis of solving Laplacian related applications on net of (regular) hexagons may be based on series method (Lee, Tien, Luo \& Luk, 2014), M-matrix theory (Lee, Tien, Luo \& Luk, 2014) and (Lee, August 2017), functional interpolation (Lee, 2015), or Fourier vectors (Lee, August 2017). The current work discusses the Laplacian through discrete symmetric boundary condition (Sections 3 and 4).

In the world of differential equations, for example (Strauss, 2008), the term symmetric boundary condition is defined so as to make a (real) operator symmetric. On the other hand, as a practice for long time in the engineering literatures, the phrase symmetric boundary condition may mean, differently, that the computational domain is reduced by halving and the numerical BC on the virtual separating edge is of homogeneous Neumann type : (Kim \& Huh, 2000), (Xu \& Soares, 2013), and (Pal, Lan, Li, Hirleman \& Ma, 2015). Same as such with spherical (reflexive) symmetric boundary condition in similar situations.

We are with the operator-theoretic view (Eqs. (5, 20 and 24) in current work).

As for the remaining sections, symmetric boundary condition for the Laplacian is introduced for smooth scalar functions in Section 2, with detailed discussion in Section 3. The theory is generalized and simplified in Section 4 with simple assumption and argument. Discussed in Section 5 are many examples of symmetric boundary condition for Laplacian, including non-product type pairs (generators) for an invariant subspace of some operator on type I Hex grid. 


\section{Function Symmetric Boundary Condition for Laplacian}

We focus here on standard Laplacian (Eq. (3) with $D_{1}=D_{2}=1$ ) and recall the Green's first and second identities,

$$
\begin{aligned}
-\iint_{\Omega} f(x, y) \nabla^{2} g(x, y) d x d y & =\iint_{\Omega} \nabla f(x, y) \nabla g(x, y) d x d y-\int_{\partial \Omega} f \frac{\partial g}{\partial \vec{n}} d \gamma(t) \\
-\iint_{\Omega} g(x, y) \nabla^{2} f(x, y) d x d y & =\iint_{\Omega} \nabla f(x, y) \nabla g(x, y) d x d y-\int_{\partial \Omega} g \frac{\partial f}{\partial \vec{n}} d \gamma(t) \\
\iint_{\Omega}\left(f \nabla^{2} g-g \nabla^{2} f\right) d x d y & =\int_{\partial \Omega}\left(f \frac{\partial g}{\partial \vec{n}}-g \frac{\partial f}{\partial \vec{n}}\right) d \gamma
\end{aligned}
$$

in which $\Omega$ is a domain with $\partial \Omega$ its piecewise smooth boundary such that these formulas are valid. The outward normal on the boundary is denoted by $\partial \vec{n}$.

Definition 1. A pair of (distinct) functions $f, g \in C^{2}(\bar{\Omega})$ satisfies symmetric boundary condition (for the Laplacian), if

$$
\int_{\partial \Omega}\left(f \frac{\partial g}{\partial \vec{n}}-g \frac{\partial f}{\partial \vec{n}}\right) d \gamma=0,
$$

so that the Laplacian is symmetric on $f$ and $g,\left\langle f, \nabla^{2} g\right\rangle=\left\langle\nabla^{2} f, g\right\rangle$.

If every pair in a family of functions satisfies the symmetric boundary condition, we say the Laplacian is symmetric on the family.

Notice that, with or without satisfication of the symmetric boundary condition, we may consider symmetrization of the energy product,

$$
\langle f, g\rangle_{L}:=\frac{1}{2}\left(\left\langle f,-\nabla^{2} g\right\rangle+\left\langle g,-\nabla^{2} f\right\rangle\right)=\iint_{\Omega} \nabla f(x, y) \nabla g(x, y) d x d y-B_{2}(f, g)
$$

in which the boundary functional is

$$
B_{2}(f, g):=\frac{1}{2} \int_{\partial \Omega} \frac{\partial}{\partial \vec{n}}(f g) d \gamma(t)=\frac{1}{2} \int_{\partial \Omega}\left(f \frac{\partial g}{\partial \vec{n}}+g \frac{\partial f}{\partial \vec{n}}\right) d \gamma(t)
$$

Assuming the symmetric boundary condition, there may exist simplification of the boundary functional and the energy product.

Example 1. Some particular cases assume

$$
\partial \Omega=: \Gamma=\Gamma_{D} \cup \Gamma_{N}, \quad \Gamma_{D} \cap \Gamma_{N}=\phi,
$$

then the symmetric B.C. is satisfied, provided that

$$
\left.f\right|_{\Gamma_{D}}=\left.g\right|_{\Gamma_{D}}=\left.\frac{\partial f}{\partial \vec{n}}\right|_{\Gamma_{N}}=\left.\frac{\partial g}{\partial \vec{n}}\right|_{\Gamma_{N}}=0
$$

Accordingly, the energy product is

$$
\left\langle f,-\nabla^{2} g\right\rangle=\left\langle g,-\nabla^{2} f\right\rangle=\iint_{\Omega} \nabla f(x, y) \nabla g(x, y) d x d y
$$

Two classical examples include pairs both satisfying homogeneous Dirichlet or homogeneous Neumann BC.

Example 2. A pair satisfying Robin $B C$ that

$$
\frac{\partial f}{\partial \vec{n}}(x)=-c(x) f(x), \quad \frac{\partial g}{\partial \vec{n}}(x)=-c(x) g(x)
$$

provides another example which satisfies the symmetric boundary condition, owing to

$$
\int_{\partial \Omega} f \frac{\partial g}{\partial \vec{n}} d \gamma(t)=-\int_{\partial \Omega} c(x(t)) f(x(t)) g(x(t)) d \gamma(t)=\int_{\partial \Omega} g \frac{\partial f}{\partial \vec{n}} d \gamma(t),
$$


The corresponding energy product

$$
\left\langle f,-\nabla^{2} g\right\rangle=\left\langle g,-\nabla^{2} f\right\rangle=\iint_{\Omega} \nabla f(x, y) \nabla g(x, y) d x d y+\int_{\partial \Omega} c(x) f(x) g(x) d \gamma
$$

is then positive definite, under an additional assumption that $c(x)>0$ on $\partial \Omega$.

Very general results in discrete symmetric boundary condition are presented in Section 4. For the motivation, we discuss next the discretization (Fig. 1) of a Cartesian net of type I regular hexagons.

\section{Discrete Symmetric Boundary Condition on Net of Regular Hexagons}

The type I neighborhood topology (Fig. 3) and the geometry (Eq. (2)) are detailed below.

$$
\begin{aligned}
\Omega:= & \left\{(i, j) \mid 1 \leq i \leq n_{x}, 1 \leq j \leq n_{y}\right\}, \\
\partial_{i}:= & \left\{(i, j) \in \Omega \mid(i-1)(j-1)\left(i-n_{x}\right)\left(j-n_{y}\right)=0\right\}, \\
\partial_{e}:= & \left\{(0,0),\left(n_{x}+1, n_{y}+1\right)\right\} \\
& \cup\left\{(i, j) \mid i=0, n_{x}+1,1 \leq j \leq n_{y}\right\} \\
& \cup\left\{(i, j) \mid j=0, n_{y}+1,1 \leq i \leq n_{x}\right\}, \\
\bar{\Omega}:= & \Omega \cup \partial_{e}, \\
\bar{\Omega}_{S}:= & \{S(P), S E(P), S W(P) \mid P \in \Omega\}, \\
\bar{\Omega}_{N}:= & \{N(P), N E(P), N W(P) \mid P \in \Omega\}, \\
\partial_{S}:= & \partial_{e} \cap \bar{\Omega}_{S}, \\
\partial_{N}:= & \partial_{e} \cap \bar{\Omega}_{N},
\end{aligned}
$$

interior nodes interior boundary exterior (ghost) boundary interior and exterior non-top-most part non-lowest part southern boundary northern boundary

With $P=(i, j) \in \Omega$,

$$
\begin{aligned}
& S \equiv S(P)=(i, j-1) \\
& N \equiv N(P)=(i, j+1) \\
& S W \equiv S W(P)= \begin{cases}(i-1, j-1), & \text { with odd } i \\
(i-1, j), & \text { with even } i\end{cases} \\
& S E \equiv S E(P)= \begin{cases}(i+1, j-1), & \text { with odd } i \\
(i+1, j), & \text { with even } i\end{cases} \\
& N W \equiv N W(P)= \begin{cases}(i-1, j), & \text { with odd } i \\
(i-1, j+1), & \text { with even } i\end{cases} \\
& N E \equiv N E(P)= \begin{cases}(i+1, j), & \text { with odd } i \\
(i+1, j+1), & \text { with even } i\end{cases}
\end{aligned}
$$

For the symmetry of the negative hexagonal seven-point Laplacian

$$
\left(L_{7} f\right)_{P}:=6 f_{P}-f_{N}-f_{S}-f_{N W}-f_{S E}-f_{N E}-f_{S W}
$$

we use backward (in vertical direction) differences to derive

$$
\begin{aligned}
\left\langle L_{7} f, g\right\rangle= & \sum_{P \in \Omega}\left(g_{P}\left(f_{P}-f_{S}\right)-g_{P}\left(f_{N}-f_{P}\right)+g_{P}\left(f_{P}-f_{S W}\right)-g_{P}\left(f_{N E}-f_{P}\right)\right. \\
& \left.+g_{P}\left(f_{P}-f_{N W}\right)-g_{P}\left(f_{S E}-f_{P}\right)\right) \\
= & \sum_{P \in \Omega}\left(\left(f_{P}-f_{S}\right)\left(g_{P}-g_{S}\right)+\left(f_{P}-f_{S W}\right)\left(g_{P}-g_{S W}\right)+\left(f_{P}-f_{N W}\right)\left(g_{P}-g_{N W}\right)\right) \\
& -\sum_{N \in \partial_{e}} g_{P}\left(f_{N}-f_{P}\right)+\sum_{S \in \partial_{e}} g_{S}\left(f_{P}-f_{S}\right) \\
& -\sum_{N E \in \partial_{e}} g_{P}\left(f_{N E}-f_{P}\right)+\sum_{S W \in \partial_{e}} g_{S W}\left(f_{P}-f_{S W}\right) \\
& -\sum_{S E \in \partial_{e}} g_{P}\left(f_{S E}-f_{P}\right)+\sum_{N W \in \partial_{e}} g_{N W}\left(f_{P}-f_{N W}\right)
\end{aligned}
$$


Similarly, by interchanging $f$ and $g$,

$$
\begin{aligned}
\left\langle f, L_{7} g\right\rangle= & \sum_{P \in \Omega}\left(f_{P}\left(g_{P}-g_{S}\right)-f_{P}\left(g_{N}-g_{P}\right)+f_{P}\left(g_{P}-g_{S W}\right)-f_{P}\left(g_{N E}-g_{P}\right)\right. \\
& \left.+f_{P}\left(g_{P}-g_{N W}\right)-f_{P}\left(g_{S E}-g_{P}\right)\right) \\
= & \sum_{P \in \Omega}\left(\left(f_{P}-f_{S}\right)\left(g_{P}-g_{S}\right)+\left(f_{P}-f_{S W}\right)\left(g_{P}-g_{S W}\right)+\left(f_{P}-f_{N W}\right)\left(g_{P}-g_{N W}\right)\right) \\
& -\sum_{N \in \partial_{e}} f_{P}\left(g_{N}-g_{P}\right)+\sum_{S \in \partial_{e}} f_{S}\left(g_{P}-g_{S}\right) \\
& -\sum_{N E \in \partial_{e}} f_{P}\left(g_{N E}-g_{P}\right)+\sum_{S W \in \partial_{e}} f_{S W}\left(g_{P}-g_{S W}\right) \\
& -\sum_{S E \in \partial_{e}} f_{P}\left(g_{S E}-g_{P}\right)+\sum_{N W \in \partial_{e}} f_{N W}\left(g_{P}-g_{N W}\right)
\end{aligned}
$$

Setting the goal $\left\langle L_{7} f, g\right\rangle-\left\langle f, L_{7} g\right\rangle=0$ leads to backward difference version of the symmetric boundary condition,

$$
\begin{aligned}
& \quad \sum_{N \in \partial_{e}}\left(f_{P}\left(g_{N}-g_{P}\right)-g_{P}\left(f_{N}-f_{P}\right)\right)-\sum_{S \in \partial_{e}}\left(f_{S}\left(g_{P}-g_{S}\right)-g_{S}\left(f_{P}-f_{S}\right)\right) \\
& +\sum_{N E \in \partial_{e}}\left(f_{P}\left(g_{N E}-g_{P}\right)-g_{P}\left(f_{N E}-f_{P}\right)\right)-\sum_{S W \in \partial_{e}}\left(f_{S W}\left(g_{P}-g_{S W}\right)-g_{S W}\left(f_{P}-f_{S W}\right)\right) \\
& +\sum_{S E \in \partial_{e}}\left(f_{P}\left(g_{S E}-g_{P}\right)-g_{P}\left(f_{S E}-f_{P}\right)\right)-\sum_{N W \in \partial_{e}}\left(f_{N W}\left(g_{P}-g_{N W}\right)-g_{N W}\left(f_{P}-f_{N W}\right)\right) \\
& =0
\end{aligned}
$$

Alternatively, we can make use of forward (in vertical direction) differences.

$$
\begin{aligned}
\left\langle L_{7} f, g\right\rangle= & \sum_{P \in \Omega}\left(\left(f_{N}-f_{P}\right)\left(g_{N}-g_{P}\right)+\left(f_{N E}-f_{P}\right)\left(g_{N E}-g_{P}\right)+\left(f_{S E}-f_{P}\right)\left(g_{S E}-g_{P}\right)\right) \\
& -\sum_{N \in \partial_{e}} g_{N}\left(f_{N}-f_{P}\right)+\sum_{S \in \partial_{e}} g_{P}\left(f_{P}-f_{S}\right) \\
& -\sum_{N E \epsilon \partial_{e}} g_{N E}\left(f_{N E}-f_{P}\right)+\sum_{S W \epsilon \partial_{e}} g_{P}\left(f_{P}-f_{S W}\right) \\
& -\sum_{S E \epsilon \partial_{e}} g_{S E}\left(f_{S E}-f_{P}\right)+\sum_{N W \epsilon \partial_{e}} g_{P}\left(f_{P}-f_{N W}\right)
\end{aligned}
$$

Also, by interchanging $f$ and $g$,

$$
\begin{aligned}
\left\langle f, L_{7} g\right\rangle= & \sum_{P \in \Omega}\left(\left(f_{N}-f_{P}\right)\left(g_{N}-g_{P}\right)+\left(f_{N E}-f_{P}\right)\left(g_{N E}-g_{P}\right)+\left(f_{S E}-f_{P}\right)\left(g_{S E}-g_{P}\right)\right) \\
& -\sum_{N \in \partial_{e}} f_{N}\left(g_{N}-g_{P}\right)+\sum_{S \in \partial_{e}} f_{P}\left(g_{P}-g_{S}\right) \\
& -\sum_{N E \in \partial_{e}} f_{N E}\left(g_{N E}-g_{P}\right)+\sum_{S W \in \partial_{e}} f_{P}\left(g_{P}-g_{S W}\right) \\
& -\sum_{S E \in \partial_{e}} f_{S E}\left(g_{S E}-g_{P}\right)+\sum_{N W \in \partial_{e}} f_{P}\left(g_{P}-g_{N W}\right)
\end{aligned}
$$

Comparison of the last two equations leads to forward difference version of the symmetric boundary condition

$$
\begin{aligned}
& \sum_{N \in \partial_{e}}\left(f_{N}\left(g_{N}-g_{P}\right)-g_{N}\left(f_{N}-f_{P}\right)\right)-\sum_{S \in \partial_{e}}\left(f_{P}\left(g_{P}-g_{S}\right)-g_{P}\left(f_{P}-f_{S}\right)\right) \\
+ & \sum_{N E \epsilon \partial_{e}}\left(f_{N E}\left(g_{N E}-g_{P}\right)-g_{N E}\left(f_{N E}-f_{P}\right)\right)-\sum_{S W \in \partial_{e}}\left(f_{P}\left(g_{P}-g_{S W}\right)-g_{P}\left(f_{P}-f_{S W}\right)\right) \\
+ & \sum_{S E \in \partial_{e}}\left(f_{S E}\left(g_{S E}-g_{P}\right)-g_{S E}\left(f_{S E}-f_{P}\right)\right)-\sum_{N W \in \partial_{e}}\left(f_{P}\left(g_{P}-g_{N W}\right)-g_{P}\left(f_{P}-f_{N W}\right)\right) \\
= & 0
\end{aligned}
$$

Taking average of Eqs. (10 and 13), (11 and 14), respectively, leads to

$$
\begin{aligned}
\left\langle L_{7} f, g\right\rangle= & \frac{1}{2} \quad \sum_{P \in \Omega}\left(\left(f_{P}-f_{S}\right)\left(g_{P}-g_{S}\right)+\left(f_{P}-f_{S W}\right)\left(g_{P}-g_{S W}\right)+\left(f_{P}-f_{N W}\right)\left(g_{P}-g_{N W}\right)\right) \\
+\frac{1}{2} & \sum_{P \in \Omega}\left(\left(f_{N}-f_{P}\right)\left(g_{N}-g_{P}\right)+\left(f_{N E}-f_{P}\right)\left(g_{N E}-g_{P}\right)+\left(f_{S E}-f_{P}\right)\left(g_{S E}-g_{P}\right)\right) \\
& -\sum_{N \in \partial_{e}} \frac{\left(g_{N}+g_{P}\right)}{2}\left(f_{N}-f_{P}\right)+\sum_{S \in \partial_{e}} \frac{\left(g_{P}+g_{S}\right)}{2}\left(f_{P}-f_{S}\right) \\
& -\sum_{N E \in \partial_{e}} \frac{\left(g_{N E}+g_{P}\right)}{2}\left(f_{N E}-f_{P}\right)+\sum_{S W \in \partial_{e}} \frac{\left(g_{P}+g_{S W}\right)}{2}\left(f_{P}-f_{S W}\right) \\
& -\sum_{S E \in \partial_{e}} \frac{\left(g_{S E}+g_{P}\right)}{2}\left(f_{S E}-f_{P}\right)+\sum_{N W \in \partial_{e}} \frac{\left(g_{P}+g_{N W}\right)}{2}\left(f_{P}-f_{N W}\right)
\end{aligned}
$$




$$
\begin{aligned}
\left\langle f, L_{7} g\right\rangle= & \frac{1}{2} \quad \sum_{P \in \Omega}\left(\left(f_{P}-f_{S}\right)\left(g_{P}-g_{S}\right)+\left(f_{P}-f_{S W}\right)\left(g_{P}-g_{S W}\right)+\left(f_{P}-f_{N W}\right)\left(g_{P}-g_{N W}\right)\right) \\
+\frac{1}{2} & \sum_{P \in \Omega}\left(\left(f_{N}-f_{P}\right)\left(g_{N}-g_{P}\right)+\left(f_{N E}-f_{P}\right)\left(g_{N E}-g_{P}\right)+\left(f_{S E}-f_{P}\right)\left(g_{S E}-g_{P}\right)\right) \\
& -\sum_{N \in \partial_{e}} \frac{\left(f_{N}+f_{P}\right)}{2}\left(g_{N}-g_{P}\right)+\sum_{S \in \partial_{e}} \frac{\left(f_{P}+f_{S}\right)}{2}\left(g_{P}-g_{S}\right) \\
& -\sum_{N E \in \partial_{e}} \frac{\left(f_{N E}+f_{P}\right)}{2}\left(g_{N E}-g_{P}\right)+\sum_{S W \in \partial_{e}} \frac{\left(f_{P}+f_{S W}\right)}{2}\left(g_{P}-g_{S W}\right) \\
& -\sum_{S E \in \partial_{e}} \frac{\left(f_{S E}+f_{P}\right)}{2}\left(g_{S E}-g_{P}\right)+\sum_{N W \in \partial_{e}} \frac{\left(f_{P}+f_{N W}\right)}{2}\left(g_{P}-g_{N W}\right)
\end{aligned}
$$

To summarize in compact form. Let $P^{\prime}$ represent a member in the neighborhood of $P \in \Omega$, that is, symbolically,

$$
P^{\prime} \in\left\{P_{N}, P_{N W}, P_{S W}, P_{S}, P_{S E}, P_{N E}\right\}
$$

Then, in terms of backward differences (using three lower neighbors, Eq. (10)),

$$
\left\langle L_{7} f, g\right\rangle=\sum_{P^{\prime} \in \Omega_{S}}\left(g_{P}-g_{P^{\prime}}\right)\left(f_{P}-f_{P^{\prime}}\right)+\sum_{P^{\prime} \in \partial_{S}} g_{P^{\prime}}\left(f_{P}-f_{P^{\prime}}\right)-\sum_{P^{\prime} \in \partial_{N}} g_{P}\left(f_{P^{\prime}}-f_{P}\right)
$$

and, in terms of forward differences (using three upper neighbors, Eq. (13)),

$$
\left\langle L_{7} f, g\right\rangle=\sum_{P^{\prime} \in \Omega_{N}}\left(g_{P^{\prime}}-g_{P}\right)\left(f_{P^{\prime}}-f_{P}\right)+\sum_{P^{\prime} \in \partial_{S}} g_{P}\left(f_{P}-f_{P^{\prime}}\right)-\sum_{P^{\prime} \in \partial_{N}} g_{P^{\prime}}\left(f_{P^{\prime}}-f_{P}\right)
$$

Taking average of these two leads to a compact form of Eq. (16),

$$
\left\langle L_{7} f, g\right\rangle=\frac{1}{2} \sum_{P \in \Omega}\left(g_{P}-g_{P^{\prime}}\right)\left(f_{P}-f_{P^{\prime}}\right)-\sum_{P^{\prime} \in \partial_{e}} \frac{g_{P}+g_{P^{\prime}}}{2}\left(f_{P^{\prime}}-f_{P}\right)
$$

Similarly, with $f$ and $g$ interchanged, we obtain compact forms of Eqs. (11 and 14),

$$
\begin{aligned}
& \left\langle f, L_{7} g\right\rangle=\sum_{P^{\prime} \in \Omega_{S}}\left(f_{P}-f_{P^{\prime}}\right)\left(g_{P}-g_{P^{\prime}}\right)+\sum_{P^{\prime} \in \partial_{S}} f_{P^{\prime}}\left(g_{P}-g_{P^{\prime}}\right)-\sum_{P^{\prime} \in \partial_{N}} f_{P}\left(g_{P^{\prime}}-g_{P}\right) \\
& \left\langle f, L_{7} g\right\rangle=\sum_{P^{\prime} \in \Omega_{N}}\left(f_{P^{\prime}}-f_{P}\right)\left(g_{P^{\prime}}-g_{P}\right)+\sum_{P^{\prime} \in \partial_{S}} f_{P}\left(g_{P}-g_{P^{\prime}}\right)-\sum_{P^{\prime} \in \partial_{N}} f_{P^{\prime}}\left(g_{P^{\prime}}-g_{P}\right)
\end{aligned}
$$

and their average, now as a compact form of Eq. (17),

$$
\left\langle f, L_{7} g\right\rangle=\frac{1}{2} \sum_{P \in \Omega}\left(f_{P}-f_{P^{\prime}}\right)\left(g_{P}-g_{P^{\prime}}\right)-\sum_{P^{\prime} \in \partial_{e}} \frac{f_{P}+f_{P^{\prime}}}{2}\left(g_{P^{\prime}}-g_{P}\right)
$$

In summary.

Theorem 2 (Symmetric boundary condition and energy product for negative seven-point Laplacian on Cartesian net of type I regular hexagons.)

$$
\begin{aligned}
\left\langle g, L_{7} f\right\rangle-\left\langle f, L_{7} g\right\rangle=0 & \Leftrightarrow \quad \sum_{P^{\prime} \in \partial_{e}}\left(\frac{\left(f_{P}+f_{P^{\prime}}\right)}{2}\left(g_{P^{\prime}}-g_{P}\right)-\frac{\left(g_{P}+g_{P^{\prime}}\right)}{2}\left(f_{P^{\prime}}-f_{P}\right)\right)=0 \\
& \Leftrightarrow \sum_{P^{\prime} \in \partial_{e}}\left(f_{P} g_{P^{\prime}}-f_{P^{\prime}} g_{P}\right)=0
\end{aligned}
$$

If the above (implied) symmetric boundary condition is satisfied, then the bilinear form

$$
\langle f, g\rangle_{L_{7}}:=\left\langle L_{7} f, g\right\rangle=\left\langle f, L_{7} g\right\rangle=\frac{1}{2} \sum_{P \in \Omega}\left(f_{P^{\prime}}-f_{P}\right)\left(g_{P^{\prime}}-g_{P}\right)+\frac{1}{2} \sum_{P^{\prime} \in \partial_{e}}\left(f_{P} g_{P}-f_{P^{\prime}} g_{P^{\prime}}\right)
$$

is well-defined and symmetric.

Proof. Taking difference of Eqs. (19 and 18) yields the symmetric boundary condition, while taking average leads to the discrete product.

Remark 1. The Theorem is valid on a general (not necessarily Cartesian) net of type I hexagons, because there is no 
usage of integral indices in the relevant discussion. Even more general case is applicable with central differencing in a proper setup, as presented in Section 4.

Example 3. (Stencil-truncation.) Assume cell-average Dirichlet BC that the grid data vanish at ghost nodes, $f_{P^{\prime}}=g_{P^{\prime}}=$ $0, \forall P^{\prime} \in \partial_{e}$, the symmetric boundary condition (Eq. (20)) is then satisfied. The simplified energy product

$$
\langle f, g\rangle_{L_{7}}=\frac{1}{2} \sum_{P \in \Omega}\left(f_{P^{\prime}}-f_{P}\right)\left(g_{P^{\prime}}-g_{P}\right)+\frac{1}{2} \sum_{P \in \partial_{i}} f_{P} g_{P}
$$

is certainly positive-definite. We note the boundary functional is defined with multiplicities at interior boundary nodes.

Example 4. (Torus.) A Cartesian net of hexagons (Fig. 1), as a computational domain with periodic BC, corresponds to a torus with no exterior boundary (ghost) node. The symmetric boundary condition for Laplacian is satisfied. The boundary functional vanishes and the discrete energy product is positive semi-definite. $\langle f, f\rangle_{L_{7}}=0$ if and only if $f$ is a (single) constant with the discrete topology being path-connected.

There are more examples along this line, with the periodic BC replaced by twist $\mathrm{BC}$ or by mixing of periodic and twist, resulting in applications of dynamical systems for the real projective plane or a Klein bottle. Open field problems subject to homogeneous Dirichlet or Neumann BC are discussed as corollaries to the general result in the next section.

\section{Symmetric Boundary Condition for Discrete Laplacian by Cell-center Finite Difference}

For applications involving discrete (negative) Laplacian in the form

$$
L(f):=\sum_{P^{\prime} \in \mathcal{N}(P)} A_{P^{\prime}}\left(f_{P}-f_{P^{\prime}}\right),
$$

with $\Omega$ and $\partial_{e}$ denoting respectively the (disjoint) set of interior grid nodes and ghost nodes, we assume very general assumptions that

(i) the discrete neighborhood topology is reflexive so that being-neighbor-to is a symmetric relation among interior nodes,

$$
P, Q \in \Omega, Q \in \mathcal{N}(P) \Longleftrightarrow P \in \mathcal{N}(Q),
$$

(ii) the central difference (CD) stencil is symmetric, assuming proper orientation (ordering) consistently in all (local) neighbor lists, such that

$$
\begin{array}{rlr}
A & :=\left\{A_{P^{\prime}} \mid P^{\prime} \in \mathcal{N}(P)\right\} & \text { (independent of } P \in \Omega \text { ) } \\
& \equiv\left\{A(P, Q) \mid P \in \Omega, Q \in \mathcal{N}(P) \subset \Omega \cap \partial_{e}\right\}, & A(P, Q)=A(Q, P) \text { if } P, Q \in \Omega .
\end{array}
$$

As an example, consider standard Laplacian on a net of type I regular hexagons (Figs. 1 and 3, and Eq. (3)), the homogeneous discrete neighborhood is the relation

$$
\mathcal{N}:=\{(P, N(P)),(P, N W(P)),(P, S W(P)),(P, S(P)),(P, S E(P)),(P, N E(P))\} \subset \Omega \times\left(\Omega \cup \partial_{e}\right)
$$

which is symmetric among interior nodes. We abuse the notation slightly and use

$$
\mathcal{N}(P) \equiv\{N(P), N W(P), S W(P), S(P), S E(P), N E(P)\}
$$

or, with implied dependence,

$$
\mathcal{N}=\{N, N W, S W, S, S E, N E\}, \quad \forall P \in \Omega .
$$

The consistently ordered homogeneous symmetric stencil (Lee,2015) is,

$$
A:=\left\{A_{N}, A_{N W}, A_{S W}, A_{S}, A_{S E}, A_{N E}\right\}=\left\{\frac{1}{6}, \frac{1}{6}, \frac{1}{6}, \frac{1}{6}, \frac{1}{6}, \frac{1}{6}\right\}, \quad \forall P \in \Omega,
$$

and, in order,

$$
\begin{aligned}
& \forall P \in \Omega, \quad P^{\prime} \in\{N, N W, S W, S, S E, N E\} \\
& A_{P^{\prime}} \in\left\{A_{N}, A_{N W}, A_{S W}, A_{S}, A_{S E}, A_{N E}\right\}=\left\{\frac{1}{6}, \frac{1}{6}, \frac{1}{6}, \frac{1}{6}, \frac{1}{6}, \frac{1}{6}\right\} .
\end{aligned}
$$


We add in passing, for anisotropic negative Laplacian (Eq. (3) on type I Hex net, that (Lee,2015)

$$
A \sim\left\{3 D_{2}-D_{1}, 2 D_{1}, 2 D_{1}, 3 D_{2}-D_{1}, 2 D_{1}, 2 D_{1}\right\}
$$

The following relations are helpful.

\section{Lemma 3}

$$
\begin{aligned}
& \sum_{P \in \Omega} \sum_{P^{\prime} \in \mathcal{N}(P) \cap \Omega} A_{P^{\prime}}\left(f_{P} g_{P^{\prime}}-f_{P^{\prime}} g_{P}\right)=0 \\
& \sum_{P \in \Omega} \sum_{P^{\prime} \in \mathcal{N}(P) \cap \Omega} A_{P^{\prime}}\left(f_{P} g_{P}-f_{P^{\prime}} g_{P^{\prime}}\right)=0
\end{aligned}
$$

We establish a very general result, as follows.

Theorem 4 The symmetric boundary condition for $\langle L f, g\rangle=\langle L g, f\rangle$ is

$$
\sum_{P \in \Omega, P^{\prime} \in \mathcal{N}(P) \cap \partial_{e}} A_{P^{\prime}}\left(f_{P} g_{P^{\prime}}-f_{P^{\prime}} g_{P}\right)=0
$$

and up to satisfication of which, the resulting energy product is

$$
\langle f, g\rangle_{L}=\sum_{P \in \Omega} \sum_{P^{\prime} \in \mathcal{N}(P)} A_{P^{\prime}}\left(f_{P^{\prime}}-f_{P}\right)\left(g_{P^{\prime}}-g_{P}\right)+\sum_{P \in \Omega, P^{\prime} \in \mathcal{N}(P) \cap \partial_{e}}\left(f_{P} g_{P}-f_{P^{\prime}} g_{P^{\prime}}\right)
$$

Proof. Note that

$$
\begin{aligned}
\langle L f, g\rangle & =\sum_{P \in \Omega} \sum_{P^{\prime} \in \mathcal{N}(P)} A_{P^{\prime}}\left(f_{P}-f_{P^{\prime}}\right) g_{P} \\
& =\sum_{P \in \Omega} \sum_{P^{\prime} \in \mathcal{N}(P) \cap \Omega} A_{P^{\prime}}\left(f_{P}-f_{P^{\prime}}\right) g_{P}+\sum_{P \in \Omega} \sum_{P^{\prime} \in \mathcal{N}(P) \cap \partial_{e}} A_{P^{\prime}}\left(f_{P}-f_{P^{\prime}}\right) g_{p} \\
\langle L g, f\rangle & =\sum_{P \in \Omega} \sum_{P^{\prime} \in \mathcal{N}(P) \cap \Omega} A_{P^{\prime}}\left(g_{P}-g_{P^{\prime}}\right) f_{P}+\sum_{P \in \Omega} \sum_{P^{\prime} \in \mathcal{N}(P) \cap \partial_{e}} A_{P^{\prime}}\left(g_{P}-g_{P^{\prime}}\right) f_{P}
\end{aligned}
$$

By taking difference of the last two equations and using Eq. (22), we obtain

$$
\begin{aligned}
\langle L f, g\rangle-\langle L g, f\rangle & =\sum_{P \in \Omega} \sum_{P^{\prime} \in \mathcal{N}(P) \cap \partial_{e}} A_{P^{\prime}}\left(f_{P^{\prime}} g_{P^{\prime}}-f_{P^{\prime}} g_{P}\right) \\
\text { (also) } \quad & =\sum_{P \in \Omega} \sum_{P^{\prime} \in \mathcal{N}(P) \cap \partial_{e}} A_{P^{\prime}}\left(\frac{f_{P^{\prime}}+f_{P}}{2}\left(g_{P^{\prime}}-g_{P}\right)-\frac{g_{P^{\prime}}+g_{P}}{2}\left(f_{P^{\prime}}-f_{P}\right)\right)
\end{aligned}
$$

which yields the symmetric boundary condition (Eq. (24)), while taking the average and using Eq. (23),

$$
\begin{aligned}
\langle f, g\rangle_{L} & :=\frac{1}{2}(\langle L f, g\rangle+\langle L g, f\rangle)=\langle L f, g\rangle=\langle L g, f\rangle \\
& =\frac{1}{2} \sum_{P \in \Omega} \sum_{P^{\prime} \in \mathcal{N}(P) \cap \Omega} A_{P^{\prime}}\left(f_{P} g_{P}+f_{P^{\prime}} g_{P^{\prime}}-f_{P} g_{P^{\prime}}-f_{P^{\prime}} g_{P}\right)+\frac{1}{2} \sum_{P \in \Omega} \sum_{P^{\prime} \in \mathcal{N}(P) \cap \in \partial_{e}} A_{P^{\prime}}\left(2 f_{P} g_{P}-f_{P} g_{P^{\prime}}-f_{P^{\prime}} g_{P}\right) \\
& =\frac{1}{2} \sum_{P \in \Omega} \sum_{P^{\prime} \in \mathcal{N}(P) \cap\left(\Omega \cup \partial_{e}\right)} A_{P^{\prime}}\left(f_{P^{\prime}}-f_{P}\right)\left(g_{P^{\prime}}-g_{P}\right)-\frac{1}{2} \sum_{P \in \Omega} \sum_{P^{\prime} \in \mathcal{N}(P) \cap \partial_{e}} A_{P^{\prime}}\left(\left(f_{P^{\prime}}-f_{P}\right)\left(g_{P^{\prime}}-g_{P}\right)-2 f_{P} g_{P}+f_{P} g_{P^{\prime}}+f_{P^{\prime}} g_{P}\right) \\
& =\frac{1}{2} \sum_{P \in \Omega} \sum_{P^{\prime} \in \mathcal{N}(P)} A_{P^{\prime}}\left(f_{P^{\prime}}-f_{P}\right)\left(g_{P^{\prime}}-g_{P}\right)+B(f, g)
\end{aligned}
$$

with boundary functional

$$
B(f, g):=\frac{1}{2} \sum_{P \in \Omega, P^{\prime} \in \mathcal{N}(P) \cap \partial_{e}}\left(f_{P} g_{P}-f_{P^{\prime}} g_{P^{\prime}}\right)
$$

Thus ends the proof. 
Remark 2. A second-order central difference approximation to the contour integral term (Eq. (5)) predicts correctly the discrete symmetric boundary condition (Eqs. (26 and 20)).

Corollary 5 Suppose every exterior (ghost) boundary node, $P^{\prime} \in \partial_{e}$, is of homogeneous Dirichlet, homogeneous Neumann or Robin type such that, respectively,

$$
\begin{array}{ll}
\text { either } f_{P}+f_{P^{\prime}}=g_{P}+g_{P^{\prime}}=0, \\
\text { or } \quad f_{P}-f_{P^{\prime}}=g_{P}-g_{P^{\prime}}=0, \\
\text { or } \quad \frac{f_{P}+f_{P^{\prime}}}{f_{P}-f_{P^{\prime}}}=\frac{g_{P}+g_{P^{\prime}}}{g_{P}-g_{P^{\prime}}}=\text { const }_{P^{\prime}}, \text { at each pair }\left(P, P^{\prime}\right),
\end{array}
$$

then the symmetric boundary condition (Eq. (24)) is satisfied.

\section{Corollary 6}

(i) If a mixed type homogeneous BC consists of at least one Dirichlet and some Neumann node(s) with vanishing boundary functional (owing to $f_{P^{\prime}} / f_{P}=f_{P^{\prime}} / f_{P}= \pm 1$ at boundary), then the energy product simplifies to

$$
\langle f, g\rangle_{L}=\langle f, L g\rangle=\langle L f, g\rangle=\frac{1}{2} \sum_{P \in \Omega} \sum_{P^{\prime} \in \mathcal{N}(P)}\left(f_{P^{\prime}}-f_{P}\right)\left(g_{P^{\prime}}-g_{P}\right)
$$

and is (symmetric and) positive-definite.

(ii) If pure homogeneous Neumann BC $\left(f_{P^{\prime}}-f_{P}=g_{P^{\prime}}-g_{P}=0\right)$ holds, then the symmetric boundary condition is satisfied, the boundary functional vanishes, the energy product is reduced (to Eq. (28)) and is positive semi-definite such that $\langle f, f\rangle_{L}=0$ if and only if $f$ is constant in each (path-)connected component of $\Omega$.

(iii) In case of (function) Robin BC (Eq. (9)) with $c(x)>0$ on $\partial \Omega$, the central difference approximation

$$
\frac{f_{P^{\prime}}-f_{P}}{2 h}=-c \frac{f_{P^{\prime}}+f_{P}}{2}
$$

with $2 h$ being the node-to-node distance, implies

$$
\frac{f_{P^{\prime}}}{f_{P}}=\frac{1-c h}{1+c h}=\frac{g_{P^{\prime}}}{g_{P}}
$$

Therefore, the energy product (Eq. (25))

$$
\langle f, g\rangle_{L}=\frac{1}{2} \sum_{P \in \Omega}\left(f_{P^{\prime}}-f_{P}\right)\left(g_{P^{\prime}}-g_{P}\right)+\frac{1}{2} \sum_{\left(P, P^{\prime}\right) \in \Omega \times\left(\mathcal{N}(P) \cap \partial_{e}\right)}\left(f_{P} g_{P} \frac{4 c h}{(1+c h)^{2}}\right)
$$

is positive definite, up to $c:=c_{\left(P+P^{\prime}\right) / 2}>0$ in practice.

We note the boundary functional is defined with various multiplicities at ghost nodes.

\subsection{Symmetric Boundary Condition for Five-point Laplacian}

In a setup of rectangular grid, the scaled negative five-point Laplacian reads

$$
L f_{i, j}=4 f_{i, j}-f_{i-1, j}-f_{i+1, j}-f_{i, j-1}-f_{i, j+1},
$$

in which $1 \leq i \leq n_{x}, 1 \leq j \leq n_{y}$ at interior nodes, and $i=0, n_{x}+1, j=0, n_{y}+1$ at ghost nodes. The general theory (Eqs. (24 and 25)) specializes as follows.

Theorem 7 Let the indices run through all interior nodes, $1 \leq i \leq n_{x}, 1 \leq j \leq n_{y}$.

(i) The discrete 2D symmetric boundary condition for five-point Laplacian is

$$
\begin{aligned}
& \sum_{i}\left(f_{i, 0} g_{i, 1}-f_{i, 1} g_{i, 0}+f_{i, n_{y}+1} g_{i, n_{y}}-f_{i, n_{y}} g_{i, n_{y}+1}\right) \\
+ & \sum_{j}\left(f_{0, j} g_{1, j}-f_{1, j} g_{0, j}+f_{n_{x}+1, j} g_{n_{x}, j}-f_{n_{x}, j} g_{n_{x}+1, j}\right)=0 .
\end{aligned}
$$


(ii) Suppose the above symmetric boundary condition is satisfied, then the following expression

$$
\begin{aligned}
\langle f, g\rangle_{L}:=\langle L f, g\rangle=\langle f, L g\rangle= & \frac{1}{2} \sum_{i, j}\left(\Delta_{i} f_{i, j} \Delta_{i} g_{i, j}+\Delta_{j} f_{i, j} \triangle_{j} g_{i, j}+\nabla_{i} f_{i, j} \nabla_{i} g_{i, j}+\nabla_{j} f_{i, j} \nabla_{j} g_{i, j}\right) \\
& +\sum_{i}\left(f_{i, 1} g_{i, 1}-f_{i, 0} g_{i, 0}\right)+\sum_{i}\left(f_{i, n_{y}} g_{i, n_{y}}-f_{i, n_{y}+1} g_{i, n_{y}+1}\right) \\
& +\sum_{j}\left(f_{1, j} g_{1, j}-f_{0, j} g_{0, j}\right)+\sum_{j}\left(f_{n_{x}, j} g_{n_{x}, j}-f_{n_{x}+1, j} g_{n_{x}+1, j}\right)
\end{aligned}
$$

defines a symmetric bilinear form.

The above theory were actually studied firstly in details, and motivated the discussion of Hex grid case (Section 3) and then the very general case (Section 4). We omit any meta-analysis in deriving Eqs. (30 and 31). Instead, we specialize for a commonly encountered application on rectangular grid.

Theorem 8 (Product-form symmetric boundary condition.) Suppose that the application data are (separable) in product forms,

$$
\left(a_{i} b_{j}\right)_{i, j} \equiv\left(f_{i, j}\right)_{i, j} \neq\left(g_{i, j}\right)_{i, j} \equiv\left(c_{i} d_{j}\right)_{i, j}
$$

then the symmetric boundary condition (Eq. (30)) simplifies to

$$
\begin{array}{cl}
\left(b_{0} d_{1}-b_{1} d_{0}+b_{n_{y}+1} d_{n_{y}}-b_{n_{y}} d_{n_{y}+1}\right) & \sum_{i=1}^{n_{x}} a_{i} c_{i} \\
+\quad\left(a_{0} c_{1}-a_{1} c_{0}+a_{n_{x}+1} c_{n_{x}}-a_{n_{x}} c_{n_{x}+1}\right) & \sum_{j=1}^{n_{y}} b_{j} d_{j}=0,
\end{array}
$$

which is satisfied if any of the following four conditions holds

$$
\begin{array}{r}
b_{0} d_{1}-b_{1} d_{0}+b_{n_{y}+1} d_{n_{y}}-b_{n_{y}} d_{n_{y}+1}=a_{0} c_{1}-a_{1} c_{0}+a_{n_{x}+1} c_{n_{x}}-a_{n_{x}} c_{n_{x}+1}=0 \\
b_{0} d_{1}-b_{1} d_{0}+b_{n_{y}+1} d_{n_{y}}-b_{n_{y}} d_{n_{y}+1}=\sum_{j=1}^{n_{y}} b_{j} d_{j}=0 \\
a_{0} c_{1}-a_{1} c_{0}+a_{n_{x}+1} c_{n_{x}}-a_{n_{x}} c_{n_{x}+1}=\sum_{i=1}^{n_{x}} a_{i} c_{i}=0 \\
\sum_{i=1}^{n_{x}} a_{i} c_{i}=\sum_{j=1}^{n_{y}} b_{j} d_{j}=0
\end{array}
$$

\section{Discussion}

We give here examples of sets of (basis) vectors which all satisfy pairwisely the symmetric boundary condition for discrete Laplacian. The private $\mathrm{BC}$ in each case is indicated when it is convenient.

We consider 2D half-integral nodes

$$
x_{i}=\frac{i-0.5}{n_{x}}, \quad 1 \leq i \leq n_{x}, \quad y_{j}=\frac{j-0.5}{n_{y}}, \quad 1 \leq j \leq n_{y},
$$

with extensions to ghost nodes. Depending on applications, several Cartesian type bases exist as summarized in Table 3, in which the one-dimensional components are defined next.

\subsection{One-dimensional Fourier Vectors}

With $\left(n, k, t_{k}\right)$ denoting $\left(n_{x}, i, x_{i}\right)$ or $\left(n_{y}, j, y_{j}\right)$, the Fourier half-wave Sine and Cosine, and quarter-wave Sine and Cosine vectors are, respectively,

$$
v_{k}^{\ell}:=\sin \left(\ell \pi t_{k}\right), \quad u_{k}^{\ell}:=\cos \left((\ell-1) \pi t_{k}\right), \quad w_{k}^{\ell}:=\sin \left((\ell-0.5) \pi t_{k}\right), \quad z_{k}^{\ell}:=\cos \left((\ell-0.5) \pi t_{k}\right), \quad 1 \leq k, \ell \leq n
$$


The implied boundary values $(k=0, n)$ satisfy private BCs that

$$
v_{0}^{\ell}+v_{1}^{\ell}=v_{n+1}^{\ell}+v_{n}^{\ell}=u_{0}^{\ell}-u_{1}^{\ell}=u_{n+1}^{\ell}-u_{n}^{\ell}=w_{0}^{\ell}+w_{1}^{\ell}=w_{n+1}^{\ell}-w_{n}^{\ell}=z_{0}^{\ell}-z_{1}^{\ell}=z_{n+1}^{\ell}+z_{n}^{\ell}=0, \quad \forall \ell
$$

We allow the extension that $v_{i}^{0}=u_{i}^{n+1} \equiv 0$ and refer to Figs. 5 and 6 for several low degree instances of these vectors.

The bases defined by Eq. (38) satisfy many properties, among which we mention the following.

(1) (Central-)Even-odd symmetry in half-wave vectors.

With $f=v^{\ell}$ or $u^{\ell}, f_{n+1-i}^{\ell}=(-1)^{\ell+1} f_{i}^{\ell}$. That is,

$$
\begin{array}{ll}
v_{n+1-i}^{2 \ell-1}=v_{i}^{2 \ell-1}, & v_{n+1-i}^{2 \ell}=-v_{i}^{2 \ell}, \\
u_{n+1-i}^{2 \ell-1}=u_{i}^{2 \ell-1}, & u_{n+1-i}^{2 \ell}=-u_{i}^{2 \ell},
\end{array}
$$

In particular,

$$
\begin{array}{ll}
v_{n}^{2 \ell-1}=v_{1}^{2 \ell-1}, & v_{n}^{2 \ell}=-v_{1}^{2 \ell}, \\
u_{n}^{2 \ell-1}=u_{1}^{2 \ell-1}, & u_{n}^{2 \ell}=-u_{1}^{2 \ell},
\end{array}
$$

and therefore as twist and periodic BCs, respectively, that

$$
\left\{\begin{array} { l } 
{ v _ { n + 1 } ^ { 2 \ell - 1 } = - v _ { n } ^ { 2 \ell - 1 } = - v _ { 1 } ^ { 2 \ell - 1 } = v _ { 0 } ^ { 2 \ell - 1 } , } \\
{ u _ { n + 1 } ^ { 2 \ell } = u _ { n } ^ { 2 \ell } = - u _ { 1 } ^ { 2 \ell } = - u _ { 0 } ^ { 2 \ell } . }
\end{array} \quad \left\{\begin{array}{l}
v_{n+1}^{2 \ell}=-v_{n}^{2 \ell}=v_{1}^{2 \ell}=-v_{0}^{2 \ell}, \\
u_{n+1}^{2 \ell-1}=u_{n}^{2 \ell-1}=u_{1}^{2 \ell-1}=u_{0}^{2 \ell-1} .
\end{array}\right.\right.
$$

(2) Symmetry in quarter-wave vectors.

With $i+i^{\prime}=\ell+\ell^{\prime}=n+1$,

$$
\begin{aligned}
w_{i}^{\ell} & =(-1)^{\ell+1} z_{i^{\prime}}^{\ell}=(-1)^{i+1} z_{i}^{\ell^{\prime}}=(-1)^{\ell+1+i} w_{i^{\prime}}^{\ell^{\prime}} \\
z_{i}^{\ell} & =(-1)^{\ell+1} w_{i^{\prime}}^{\ell}=(-1)^{\ell+1} w_{i^{\prime}}^{\ell}=(-1)^{\ell+1+i} z_{i^{\prime}}^{\ell^{\prime}}
\end{aligned}
$$

(3) $1 D$ orthogonality.

$$
\begin{gathered}
\left\langle v^{\ell}, v^{p}\right\rangle=\left\{\begin{array}{rr}
n, & \ell=p=n, \\
\frac{n}{2}, & 1 \leq \ell=p<n, \\
0, & 1 \leq \ell \neq p \leq n,
\end{array} \quad\left\langle u^{\ell}, u^{p}\right\rangle= \begin{cases}n, & 1=\ell=p, \\
\frac{n}{2}, & 1<\ell=p \leq n, \\
0, & 1 \leq \ell \neq p \leq n .\end{cases} \right. \\
\left\langle w^{\ell}, w^{p}\right\rangle=\left\langle z^{\ell}, z^{p}\right\rangle=\frac{n}{2} \delta_{\ell, p}
\end{gathered}
$$

(4) $1 D$ cross-product.

$$
\begin{aligned}
& \left\langle v^{\ell}, u^{p}\right\rangle= \begin{cases}0, & \text { if } \ell-p \text { is odd } \\
\frac{1}{2}\left(\csc \frac{(\ell+p-1) \pi / 2}{n}+\csc \frac{(\ell-p+1) \pi / 2}{n}\right), & \text { if } \ell-p \text { is even. }\end{cases} \\
& \left\langle w^{\ell}, z^{p}\right\rangle=\left\{\begin{array}{cc}
-\left\langle w^{p}, z^{\ell}\right\rangle=\frac{1}{2} \csc \frac{(\ell-p) \pi}{2 n}, & \text { if } \ell-p \text { is odd, } \\
\left\langle w^{p}, z^{\ell}\right\rangle=\frac{1}{2} \csc \frac{(\ell+p-1) \pi}{2 n}, & \text { if } \ell-p \text { is even, }
\end{array}\right.
\end{aligned}
$$

Orthogonal bases exist in various forms. We state a few.

Lemma 9 Every set in the following generates an orthogonal basis of $\mathbb{R}^{n}$.
(1) $\left\{v^{\ell} \mid 1 \leq \ell \leq n\right\}$,
(3) $\left\{u^{2 \ell-1}, \quad v^{2 \ell} \mid 1 \leq \ell \leq \frac{n}{2}\right\}$,
(2) $\left\{u^{\ell} \mid 1 \leq \ell \leq n\right\}$,
(4) $\left\{v^{2 \ell-1}, \quad u^{2 \ell} \quad \mid 1 \leq \ell \leq \frac{n}{2}\right\}$. 

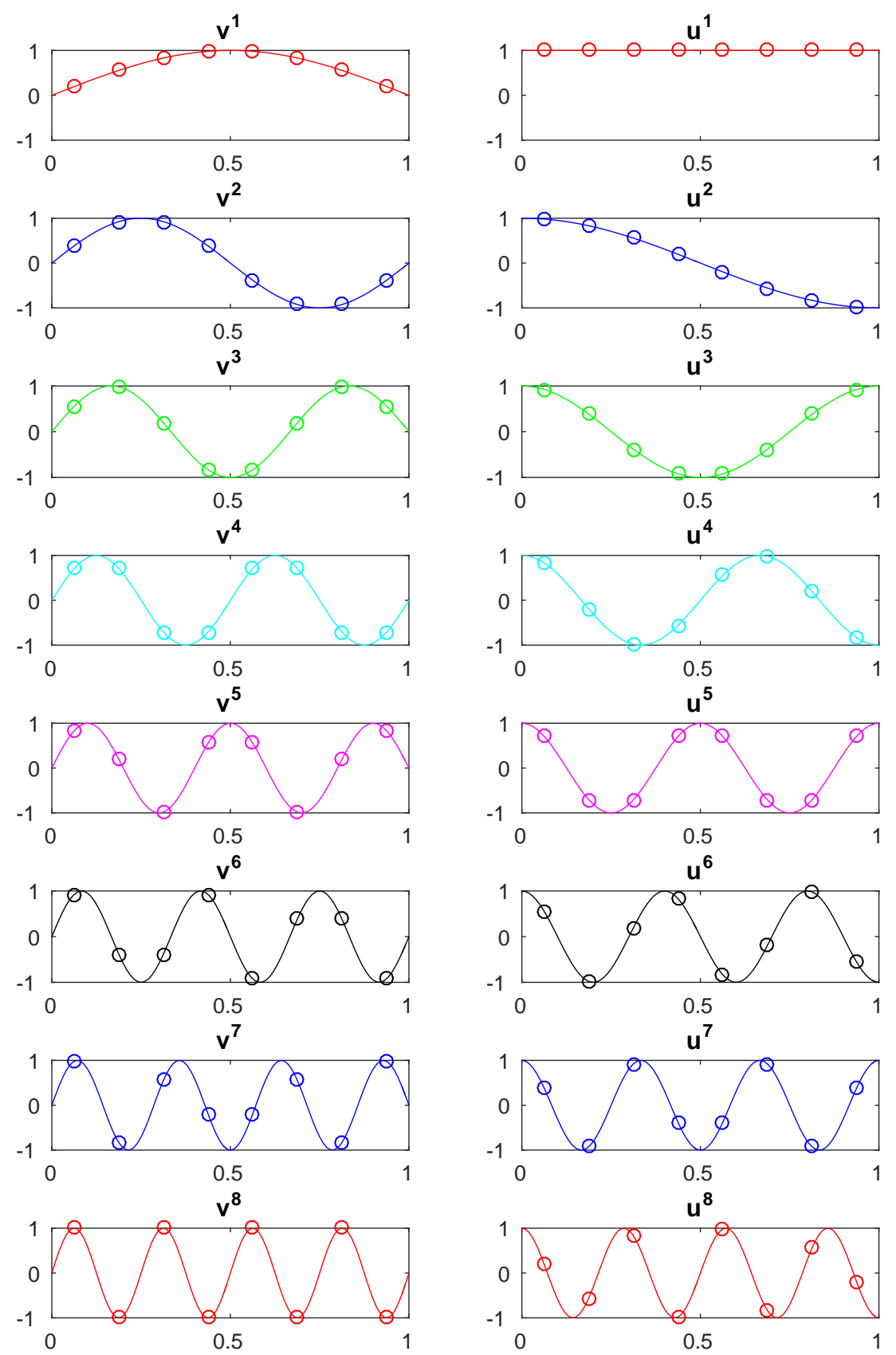

Figure 5. Fourier half-wave Sine and Cosine vectors of degrees up to eight 

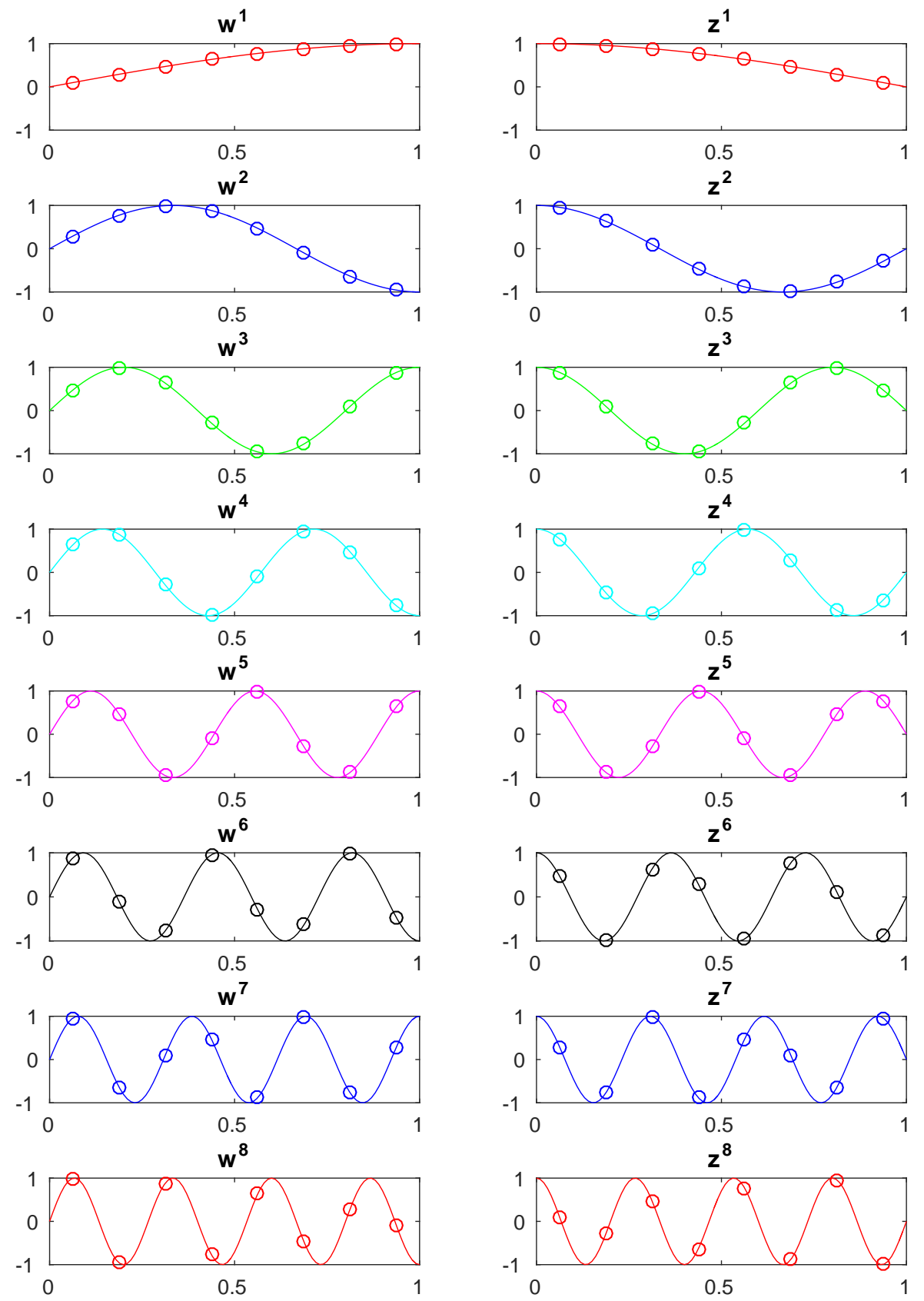

Figure 6. Fourier quarter-wave Sine and Cosine vectors of degrees up to eight 
Remark 3. These four algebraic bases are also eigenbases of the 1D three-point Laplacian subject to separable or nonseparable BCs of types (D)irichlet, (N)eumann, (P)eriodic and (T)wist, respectively, as follows.

$\begin{array}{lll}\text { (i) Dirichlet: } & f_{0}=-f_{1}, & f_{n+1}=-f_{n} \\ \text { (ii) Neumann : } & f_{0}=f_{1}, & f_{n+1}=f_{n} \\ \text { (iii) Periodic : } & f_{0}=f_{n}, & f_{n+1}=f_{1} \\ \text { (iv) Twist: } & f_{0}=-f_{n}, & f_{n+1}=-f_{1}\end{array}$

The corresponding BCs, for smooth functions, are
(i) Dirichlet: $f(0)$

$$
=f(1)
$$$$
=f^{\prime}(1)
$$$$
=0
$$
(ii) Neumann: $f^{\prime}(0)$
$f(0)-f(1)=f^{\prime}\left(0^{+}\right)-f^{\prime}\left(1^{-}\right)=0$
(iii) Periodic :
$f(0)+f(1)=f^{\prime}\left(0^{+}\right)+f^{\prime}\left(1^{-}\right)=0$

The Twist subcase suggests that the dynamics of a BVP solution be realized on a Mobius strip, while on a circle with the periodic one.

\subsection{Two-dimensional Fourier Vectors}

With admissible indices $1 \leq \ell, i \leq n_{x}, 1 \leq m, j \leq n_{y}$ and extension to ghost nodes, we define 2D Fourier Sine, Cosine and two mixed type vectors, respectively,

$$
\begin{array}{rlrl}
v^{\ell, m} & \equiv\left(v_{i, j}^{\ell, m}\right)_{i, j}, & v_{i, j}^{\ell, m}:= & v_{i}^{\ell} v_{j}^{m}=\sin \left(\ell \pi x_{i}\right) \sin \left(m \pi y_{j}\right), \\
u^{\ell, m} & \equiv\left(u_{i, j}^{\ell, m}\right)_{i, j}, & u_{i, j}^{\ell, m}:= & u_{i}^{\ell} u_{j}^{m}=\cos \left((\ell-1) \pi x_{i}\right) \cos \left((m-1) \pi y_{j}\right), \\
\left(v^{\ell} u^{m}\right)_{i, j} & \equiv\left(v_{i}^{\ell} u_{j}^{m}\right)_{i, j}, & v_{i}^{\ell} u_{j}^{m}=\sin \left(\ell \pi x_{i}\right) \cos \left((m-1) \pi y_{j}\right), \\
\left(u^{\ell} v^{m}\right)_{i, j} & \equiv\left(u_{i}^{\ell} v_{j}^{m}\right)_{i, j}, & u_{i}^{\ell} v_{j}^{m}=\cos \left((\ell-1) \pi x_{i}\right) \sin \left(m \pi y_{j}\right),
\end{array}
$$

with the convention that

$$
v^{\ell, m} \equiv 0, \text { if } \ell m=0, \quad \text { and } \quad u^{\ell, m} \equiv 0, \text { if } \ell=n_{x}+1 \text { or } m=n_{y}+1 .
$$

Another four bases of mixed type are

$$
\begin{aligned}
w^{\ell, m} & \equiv\left(w_{i, j}^{\ell, m}\right)_{i, j}, \quad w_{i, j}^{\ell, m}:=w_{i}^{\ell} w_{j}^{m}=\sin \frac{(\ell-0.5)(i-0.5) \pi}{n_{x}} \sin \frac{(m-0.5)(j-0.5) \pi}{n_{y}} \\
z^{\ell, m} & \equiv\left(z_{i, j}^{\ell, m}\right)_{i, j}, \quad z_{i, j}^{\ell, m}:=z_{i}^{\ell} z_{j}^{m}=\cos \frac{(\ell-0.5)(i-0.5) \pi}{n_{x}} \cos \frac{(m-0.5)(j-0.5) \pi}{n_{y}}
\end{aligned}
$$

and

$$
\begin{aligned}
& \left(w_{i}^{\ell} z_{j}^{m}\right)_{i, j}:=\left(\sin \frac{(\ell-0.5)(i-0.5) \pi}{n_{x}} \cos \frac{(m-0.5)(j-0.5) \pi}{n_{y}}\right)_{i, j} \\
& \left(z_{i}^{\ell} w_{j}^{m}\right)_{i, j}:=\left(\cos \frac{(\ell-0.5)(i-0.5) \pi}{n_{x}} \sin \frac{(m-0.5)(j-0.5) \pi}{n_{y}}\right)_{i, j}
\end{aligned}
$$

Appropriate BCs in applications with these bases (Eqs. (48,49 and 50)), among others, are shown in Table 3. The associated priviate $\mathrm{BCs}$ can be expressed collectively, with $1 \leq \ell \leq n_{x}, 1 \leq m \leq n_{y}$, as

$$
\begin{aligned}
& \frac{v_{0}^{\ell}}{v_{1}^{\ell}}=\frac{v_{0}^{m}}{v_{1}^{m}}=\frac{w_{0}^{\ell}}{w_{1}^{\ell}}=\frac{w_{0}^{m}}{w_{1}^{m}}=\frac{v_{n_{x}+1}^{\ell}}{v_{n_{x}}^{\ell}}=\frac{v_{n_{y}+1}^{m}}{v_{n_{y}}^{m}}=\frac{z_{n_{x}+1}^{\ell}}{z_{n_{x}}^{\ell}}=\frac{z_{n_{y}+1}^{m}}{z_{n_{y}}^{m}}=-1 \\
& \frac{u_{0}^{\ell}}{u_{1}^{\ell}}=\frac{u_{0}^{m}}{u_{1}^{m}}=\frac{z_{0}^{\ell}}{z_{1}^{\ell}}=\frac{z_{0}^{m}}{z_{1}^{m}}=\frac{u_{n_{x}+1}^{\ell}}{u_{n_{x}}^{\ell}}=\frac{u_{n_{y}+1}^{m}}{u_{n_{y}}^{m}}=\frac{w_{n_{x}+1}^{\ell}}{w_{n_{x}}^{\ell}}=\frac{w_{n_{y}+1}^{m}}{w_{n_{y}}^{m}}=1
\end{aligned}
$$

More bases and BCs appear in Tables 4 and 5, in which the types of BC are consistent with usage in Remark 3, except for a new type: $A$ (lternating between $\mathrm{D}$ and $\mathrm{N}$ ). Discrete symmetric boundary condition and energy product for five-point Laplacian are discussed next. 
Table 3. Fourier bases on rectangular half-integral nodes and associated BCs

\begin{tabular}{|c|c|c|c|c|}
\hline Basis & $\bar{B}$ & BC_e & BC_S & BC_n \\
\hline DD-DD, $\left\{\left(v_{i}^{\ell} v_{j}^{m}\right)_{i, j}\right\}$ & $v_{0}^{\ell}=-v_{1}^{\ell}$ & $v_{n_{x}+1}^{\ell}=-v_{n_{x}}^{\ell}$ & $v_{0}^{m}=-v_{1}^{m}$ & $v_{n_{y}+1}^{m}=-v_{n_{y}}^{m}$ \\
\hline NN-NN, $\left\{\left(u_{i}^{\ell} u_{j}^{m}\right)_{i, j}\right.$ & $u_{0}^{\ell}=u_{1}^{\ell}$ & $u_{n_{x}+1}^{\ell}=u_{n_{x}}^{\ell}$ & $u_{0}^{m}=u_{1}^{m}$ & $u_{n_{y}+1}^{m}=u_{n_{y}}^{m}$ \\
\hline DD-NN, $\left\{\left(v_{i}^{\ell} u_{j}^{m}\right)_{i, j}\right\}_{\ell, m}$ & $v_{0}^{\ell}=-v_{1}^{\ell}$ & $v_{n_{x}+1}^{\ell}=-v_{n_{x}}^{\ell}$ & $u_{0}^{m}=u_{1}^{m}$ & $u_{n_{y}+1}^{m}=u_{n_{y}}^{m}$ \\
\hline NN-DD, $\left\{\left(u_{i}^{\ell} v_{j}^{m}\right)_{i, j}\right\}$ & $u_{0}^{\ell}=u_{1}^{\ell}$ & $u_{n_{x}+1}^{\ell}=u_{n_{x}}^{\ell}$ & $v_{0}^{m}=-v_{1}^{m}$ & $v_{n_{y}+1}^{m}=-v_{n_{y}}^{m}$ \\
\hline DN-DN, $\left\{\left(w_{i}^{\ell} w_{j}^{m}\right)_{i, j}\right\}$ & $w_{0}^{\ell}=-w_{1}^{\ell}$ & $w_{n_{x}+1}^{\ell}=w_{n_{x}}^{\ell}$ & $w_{0}^{m}=-w_{1}^{m}$ & $w_{n_{y}+1}^{m}=w_{n_{y}}^{m}$ \\
\hline ND-ND, $\left\{\left(z_{i}^{\ell} z_{j}^{m}\right)_{i, j}\right\}_{\ell}$ & $z_{0}^{\ell}=z_{1}^{\ell}$ & $z_{n_{x}+1}^{\ell}=-z_{n_{x}}^{\ell}$ & $z_{0}^{m}=z_{1}^{m}$ & $z_{n_{y}+1}^{m}=-z_{n_{y}}^{m}$ \\
\hline DN-ND, $\left\{\left(w_{i}^{\ell} z_{j}^{m}\right)_{i, j}\right\}_{\ell}$ & $w_{0}^{\ell}=-w_{1}^{\ell}$ & $w_{n_{x}+1}^{\ell}=w_{n_{x}}^{\ell}$ & $z_{0}^{m}=z_{1}^{m}$ & $z_{n_{y}+1}^{m}=-z_{n_{y}}^{m}$ \\
\hline ND-DN, $\left\{\left(z_{i}^{\ell} w_{j}^{m}\right)_{i, j}\right\}_{\ell}$ & $z_{0}^{\ell}=z_{1}^{\ell}$ & $z_{n_{x}+1}^{\ell}=-z_{n_{x}}^{\ell}$ & $w_{0}^{m}=-w_{1}^{m}$ & $w_{n_{y}+1}^{m}=w_{n_{y}}^{m}$ \\
\hline DD-DN, $\left\{\left(v_{i}^{\ell} w_{j}^{m}\right)_{i, j}\right\}_{\ell, m}$ & $v_{0}^{\ell}=-v_{1}^{\ell}$ & $v_{n_{x}+1}^{\ell}=-v_{n_{x}}^{\ell}$ & $w_{0}^{m}=-w_{1}^{m}$ & $w_{n_{y}+1}^{m}=w_{n_{y}}^{m}$ \\
\hline DD-ND, $\left\{\left(v_{i}^{\ell} z_{j}^{m}\right)_{i, j}\right\}_{\ell, m}$ & $v_{0}^{\ell}=-v_{1}^{\ell}$ & $v_{n_{x}+1}^{\ell}=-v_{n_{x}}^{\ell}$ & $z_{0}^{m}=z_{1}^{m}$ & $z_{n_{y}+1}^{m}=-z_{n_{y}}^{m}$ \\
\hline NN-DN, $\left\{\left(u_{i}^{\ell} w_{j}^{m}\right)_{i, j}\right\}_{\ell, m}$ & $u_{0}^{\ell}=u_{1}^{\ell}$ & $u_{n_{x}+1}^{\ell}=u_{n_{x}}^{\ell}$ & $w_{0}^{m}=-w_{1}^{m}$ & $w_{n_{y}+1}^{m}=w_{n_{y}}^{m}$ \\
\hline NN-ND, $\left\{\left(u_{i}^{\ell} z_{j}^{m}\right)_{i, j}\right\}_{\ell, m}$ & $u_{0}^{\ell}=u_{1}^{\ell}$ & $u_{n_{x}+1}^{\ell}=u_{n_{x}}^{\ell}$ & $z_{0}^{m}=z_{1}^{m}$ & $z_{n_{y}+1}^{m}=-z_{n_{y}}^{m}$ \\
\hline DN-DD, $\left\{\left(w_{i}^{\ell} v_{j}^{m}\right)_{i, j}\right\}_{\ell, m}$ & $w_{0}^{\ell}=-w_{1}^{\ell}$ & $w_{n_{x}+1}^{\ell}=w_{n_{x}}^{\ell}$ & $v_{0}^{m}=-v_{1}^{m}$ & $v_{n_{y}+1}^{m}=-v_{n_{y}}^{m}$ \\
\hline DN-NN, $\left\{\left(w_{i}^{\ell} u_{j}^{m}\right)_{i, j}\right\}_{\ell, m}$ & $w_{0}^{\ell}=-w_{1}^{\ell}$ & $w_{n_{x}+1}^{\ell}=w_{n_{x}}^{\ell}$ & $u_{0}^{m}=u_{1}^{m}$ & $u_{n_{y}+1}^{m}=u_{n_{y}}^{m}$ \\
\hline ND-DD, $\left\{\left(z_{i}^{\ell} v_{j}^{m}\right)_{i, j}\right\}_{\ell, m}$ & $z_{0}^{\ell}=$ & $z_{n_{x}+1}^{\ell}=-z_{n_{x}}^{\ell}$ & $v_{0}^{m}=-v_{1}^{m}$ & $v_{n_{y}+1}^{m}=-v_{n_{y}}^{m}$ \\
\hline ND-NN, $\left\{\left(z_{i}^{\ell} u_{j}^{m}\right)_{i, j}\right\}_{\ell, m}$ & $z_{0}^{\ell}=$ & $z_{n_{x}+1}^{\ell}=-z_{n_{x}}^{\ell}$ & $u_{0}^{m}=u_{1}^{m}$ & $u_{n_{y}+1}^{m}=u_{n_{y}}^{m}$ \\
\hline
\end{tabular}


Table 4. Eigenpairs of negative 5-point Laplacian, Part I

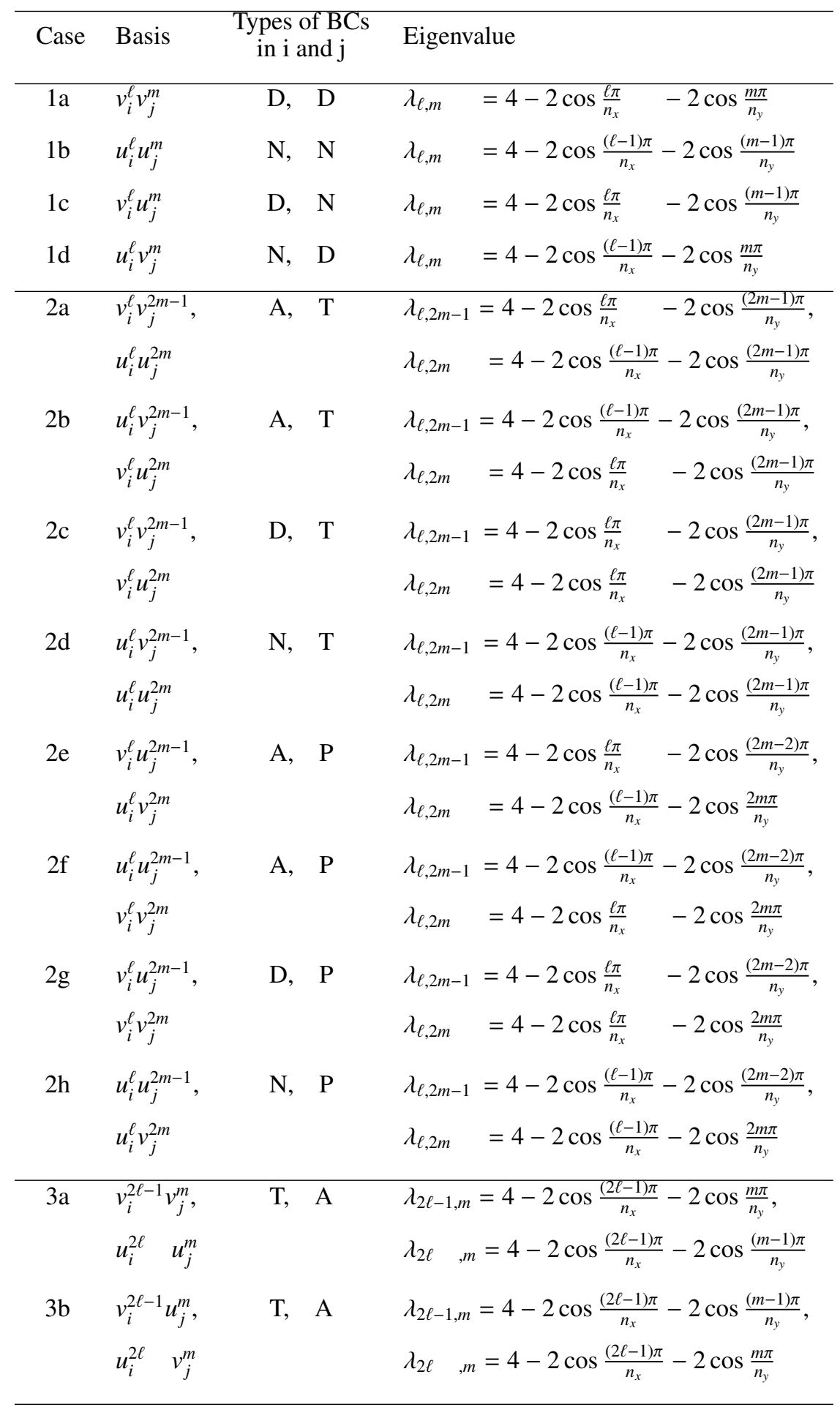


Table 5. Eigenpairs of negative 5-point Laplacian, Part II

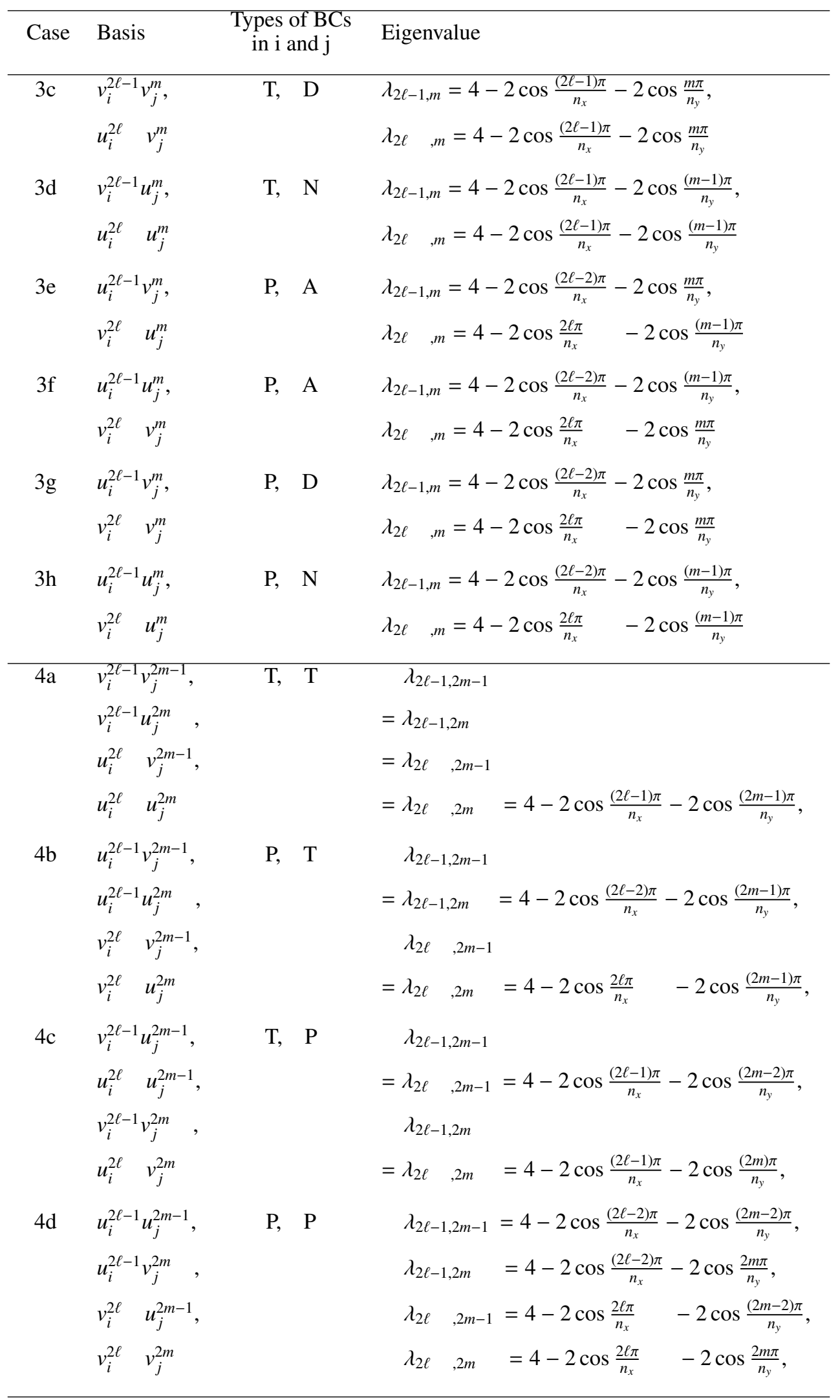




\subsection{Discrete Two-dimensional Symmetric Boundary Condition}

Following the general usage of the vector notations (Eq. (32)), we note the following.

Example 5. The product-form symmetric boundary condition (Eq. (33)) is satisfied by all the 16 bases in Table 3 .

Proof. With the specified component bases (and $f \neq g$ ), each of the individual BCs (Eq. (51)) implies

$$
\left\{\frac{a_{1}}{a_{0}}=\frac{c_{1}}{c_{0}}, \frac{a_{n_{x}+1}}{a_{n_{x}}}=\frac{c_{n_{x}+1}}{c_{n_{x}}}, \frac{b_{1}}{b_{0}}=\frac{d_{1}}{d_{0}}, \frac{b_{n_{y}+1}}{b_{n_{y}}}=\frac{d_{n_{y}+1}}{d_{n_{y}}}\right\} \subset\{ \pm 1\} .
$$

Hence the assertion, by Eq. (34).

An alternative argument exists as follows. For $a b=f \neq g=c d$,

$$
\begin{aligned}
& \text { if } a \neq c, b \neq d \text {, then }\langle a, c\rangle=\langle b, d\rangle=0, \\
& \text { if } a \neq c, b=d \text {, then }\langle a, c\rangle=b_{0} d_{1}-b_{1} d_{0}+b_{n_{y}+1} d_{n_{y}}-b_{n_{y}} d_{n_{y}+1}=0, \\
& \text { if } a=c, b \neq d \text {, then }\langle b, d\rangle=a_{0} c_{1}-a_{1} c_{0}+a_{n_{y}+1} c_{n_{y}}-a_{n_{y}} c_{n_{y}+1}=0 .
\end{aligned}
$$

All Eqs. (34, 35 and 36$)$ are thus satisfied in these 16 cases.

Discussion of cases in the next example makes use of (i) Eqs. (35 and 36), (ii) the respective BCs (Eq. (39)) of 1D Fourier vectors, and (iii) the even-odd symmetry (Eqs. (40 and 47)) that

$$
\begin{aligned}
& \left(v_{0}^{2 \ell-1}, v_{1}^{2 \ell-1}, v_{n_{x}}^{2 \ell-1}, v_{n_{x}+1}^{2 \ell-1}\right)=v_{1}^{2 \ell-1}(-1,1,1,-1), \\
& \left(v_{0}^{2 \ell}, v_{1}^{2 \ell}, v_{n_{x}}^{2 \ell}, v_{n_{x}+1}^{2 \ell}\right)=v_{1}^{2 \ell}(-1,1,-1,1), \\
& \left(u_{0}^{2 \ell-1}, u_{1}^{2 \ell-1}, u_{n_{x}}^{2 \ell-1}, u_{n_{x}+1}^{2 \ell-1}\right)=u_{1}^{2 \ell-1}(1,1,1,1), \\
& \left(u_{0}^{2 \ell}, u_{1}^{2 \ell}, u_{n_{x}}^{2 \ell}, u_{n_{x}+1}^{2 \ell}\right)=u_{1}^{2 \ell}(1,1,-1,-1),
\end{aligned}
$$

and the same holds with $\left(l, n_{x}\right)$ replaced by $\left(m, n_{y}\right)$.

Example 6. We justify the symmetric boundary condition (Eq. (33)) for all 24 bases listed in Tables 4 and 5.

(1) The first four (1a-1d) options of bases are covered in the previous example.

(2) For bases $(2 \mathrm{a}-2 \mathrm{~d})$, we note

$$
b_{j}=v_{j}^{2 m-1}, \quad d_{j}=u_{j}^{2 m}, \quad\langle b, d\rangle=\left\langle v^{2 m-1}, u^{2 m}\right\rangle=0
$$

and

$$
\begin{aligned}
\left(b_{0} d_{1},-b_{1} d_{0}, b_{n_{y}+1} d_{n_{y}},-b_{n_{y}} d_{n_{y}+1}\right) & =\left(v_{0}^{2 m-1} u_{1}^{2 m},-v_{1}^{2 m-1} u_{0}^{2 m}, v_{n_{y}+1}^{2 m-1} u_{n_{y}}^{2 m},-v_{n_{y}}^{2 m-1} u_{n_{y}+1}^{2 m}\right) \\
& =v_{1}^{2 m-1} u_{1}^{2 m}(-1,-1,1,1) .
\end{aligned}
$$

(3) For bases (2e-2h),

$$
b_{j}=u_{j}^{2 m-1}, \quad d_{j}=v_{j}^{2 m}, \quad\langle b, d\rangle=\left\langle u^{2 m-1}, v^{2 m}\right\rangle=0,
$$

and

$$
\begin{aligned}
\left(b_{0} d_{1},-b_{1} d_{0}, b_{n_{y}+1} d_{n_{y}},-b_{n_{y}} d_{n_{y}+1}\right) & =\left(u_{0}^{2 m-1} v_{1}^{2 m},-u_{1}^{2 m-1} v_{0}^{2 m}, u_{n_{y}+1}^{2 m-1} v_{n_{y}}^{2 m},-u_{n_{y}}^{2 m-1} v_{n_{y}+1}^{2 m}\right) \\
& =u_{1}^{2 m-1} v_{1}^{2 m}(1,1,-1,-1) .
\end{aligned}
$$

(4) Same argument applies to bases (3a-3h), as does for (2a-2h), by an interchange of the two symbol lists

$$
\left\{j, m, n_{y}, b, d\right\} \quad \text { and } \quad\left\{i, \ell, n_{x}, a, c\right\} .
$$


(5) It is similar for bases (4a-4d). We mention for basis set (4a), by Eq. (41), that

$$
\begin{array}{r}
f \in\left\{v_{i}^{2 \ell-1} v_{j}^{2 m-1}, \quad v_{i}^{2 \ell-1} u_{j}^{2 m}, \quad u_{i}^{2 \ell} v_{j}^{2 m-1}, \quad u_{i}^{2 \ell} u_{j}^{2 m}\right\}, \\
f_{0, j}=\epsilon f_{n_{x}, j}, \quad f_{n_{x}+1, j}=\epsilon f_{1, j}, \quad f_{i, 0}=\epsilon f_{i, n_{y}}, \quad f_{i, n_{y}+1}=\epsilon f_{i, 1} .
\end{array}
$$

with $\epsilon=-1$. Note that $\epsilon=1$ works for basis 4(d).

\subsection{Two-dimensional Non-product Type Pairs}

This is an example on type I Hex grid. For convenience, we label components of the symmetric boundary condition (Eq. (30)) as follows,

$$
\begin{aligned}
0= & 1 \text { st-sum }+2 \text { nd-sum }+3 \text { rd-sum }+4 \text { th-sum } \\
\equiv & \sum_{i=1}^{n_{x}}\left(f_{i, 0} g_{i, 1}-f_{i, 1} g_{i, 0}\right)+\sum_{i=1}^{n_{x}}\left(f_{i, n_{y}+1} g_{i, n_{y}}-f_{i, n_{y}} g_{i, n_{y}+1}\right) \\
& \quad+\sum_{j=1}^{n_{y}}\left(f_{0, j} g_{1, j}-f_{1, j} g_{0, j}\right)+\sum_{j=1}^{n_{y}}\left(f_{n_{x}+1, j} g_{n_{x}, j}-f_{n_{x}, j} g_{n_{x}+1, j}\right)
\end{aligned}
$$

and consider three non-product type families (and members),

Pair 1.

Pair 2.

$$
f, g \in\left\{z_{i}^{\ell} z_{j}^{m}+w_{i}^{\ell^{\prime}} w_{j}^{m}\right\}_{\ell, m},
$$

Pair 3.

$$
f, g \in\left\{z_{i}^{\ell} z_{j}^{m}-w_{i}^{\ell^{\prime}} w_{j}^{m}\right\}_{\ell, m},
$$

$$
f \in\left\{z_{i}^{\ell} z_{j}^{m}+w_{i}^{\ell^{\prime}} w_{j}^{m}\right\}_{\ell, m}, \quad g \in\left\{z_{i}^{\ell} z_{j}^{m}-w_{i}^{\ell^{\prime}} w_{j}^{m}\right\}_{\ell, m} .
$$

We note these are invariant under some operator, $Q_{4}^{H}$ (Lee,(2016,submitted)), on a net of type I Hex grid. In above and below, the admissible indices satisfy

$$
1 \leq i, \ell, \ell^{\prime}, p, p^{\prime} \leq n_{x}, \quad \ell+\ell^{\prime}=p+p^{\prime}=n_{x}+1, \quad 1 \leq j, m, q \leq n_{y} .
$$

Theorem 10 (Pair 1 and pair 2.) Let

$$
f_{i, j}=z_{i}^{\ell} z_{j}^{m} \pm w_{i}^{\ell^{\prime}} w_{j}^{m}, \quad g_{i, j}=z_{i}^{p} z_{j}^{q} \pm w_{i}^{p^{\prime}} w_{j}^{q} .
$$

If (i) $q=m$, or (ii) $p=\ell^{\prime}$ and $m-q$ is odd, then the symmetric boundary condition (Eq. (30)) is satisfied on both pairs.

Proof. With respect to Eq. (52), it is straight forward to derive the following,

$$
\begin{aligned}
\text { 1st-sum } & = \begin{cases} \pm \sec \frac{(\ell+p-1) \pi}{2 n_{x}} \sin \frac{(q-m) \pi}{2 n_{y}}, & \text { if } \ell-p \text { is even } \\
\pm \sec \frac{(\ell-p) \pi}{2 n_{x}} \sin \frac{(q-m) \pi}{2 n_{y}}, & \text { if } \ell-p \text { is odd }\end{cases} \\
\text { 2nd-sum } & =\begin{array}{ll}
(-1)^{q-m} \cdot(1 \mathrm{st}-\mathrm{sum}) & \text { if } q-m \text { is even }
\end{array} \\
\text { 3rd-sum } & = \begin{cases}0, & \text { if } q-m \text { is odd } \\
\pm 2 \csc \frac{(q-m) \pi}{2 n_{y}} \cos \frac{(\ell-0.5) \pi}{2 n_{x}} \cos \frac{(p-0.5) \pi}{2 n_{x}}, & \text { if } q-m \text { is even }\end{cases} \\
\text { 4th-sum } & = \begin{cases}0, & \text { if } q-m \text { is odd } \\
\pm 2 \csc \frac{(q-m) \pi}{2 n_{y}}(-1)^{\ell+p} \sin \frac{(\ell-0.5) \pi}{2 n_{x}} \sin \frac{(p-0.5) \pi}{2 n_{x}},\end{cases}
\end{aligned}
$$

This ends the proof.

Theorem 11 (Pair 3.) Let

$$
f_{i, j}=z_{i}^{\ell} z_{j}^{m}+w_{i}^{\ell^{\prime}} w_{j}^{m}, \quad g_{i, j}=z_{i}^{p} z_{j}^{q}-w_{i}^{p^{\prime}} w_{j}^{q} .
$$

If $p=\ell^{\prime}$ and $m-q$ is even, then the symmetric boundary condition (Eq. (30)) is satisfied. 
Proof. The following are obtained.

$$
\begin{aligned}
\text { 1st-sum } & = \begin{cases}-\sec \frac{(\ell+p-1) \pi}{2 n_{x}} \sin \frac{(m+q-1) \pi}{2 n_{y}}, & \text { if } \ell-p \text { is even } \\
-\sec \frac{(\ell-p) \pi}{2 n_{x}} \sin \frac{(m+q-1) \pi}{2 n_{y}}, & \text { if } \ell-p \text { is odd }\end{cases} \\
\text { 2nd-sum } & =\begin{array}{ll}
(-1)^{m-q} \cdot(1 \operatorname{st}-\operatorname{sum}) & \text { if } m-q \text { is even }
\end{array} \\
\text { 3rd-sum } & = \begin{cases}-2 \csc \frac{(m+q-1) \pi}{2 n_{y}} \cos \frac{(\ell-0.5) \pi}{2 n_{x}} \cos \frac{(p-0.5) \pi}{2 n_{x}}, & \text { if } m-q \text { is odd } \\
0, & \text { if } m-q \text { is even }\end{cases} \\
\text { 4th-sum } & = \begin{cases}(-1)^{\ell+p} 2 \csc \frac{(m+q-1) \pi}{2 n_{y}} \sin \frac{(\ell-0.5) \pi}{2 n_{x}} \sin \frac{(p-0.5) \pi}{2 n_{x}}, & \text { if } m-q \text { is odd } \\
0, & \end{cases}
\end{aligned}
$$

We note all the calculations of discrete inner-products involving finite trigonometric series are derived by hands and also verified by C-codes.

\section{Conclusions}

Cell-centered hexagonal finite volume based finite difference method were confirmed effective in Poisson problems and also in time-dependent problems such as to exhibit successfully linear and spiral waves. The hexagonal seven-point Laplacian is analyzed in this work by using symmetric boundary condition. The developed theory, together with some examples, are readily generalized to many two- and three-dimensional applications up to usage of symmetric central differences. Further generalizations to biharmonic and self-adjoint operators are expected.

\section{References}

Bystrytskyi, M. E., \& Mosklkov, M. M. (2001). The difference Laplace operator on a seven-point nonorthogonal pattern of a rectangular grid and its spectral properties. Journal of Mathematical Sciences, 104(6), 1593-1598. https://doi.org/10.1023/A:1011316910596

Van Eck, R. H. J., Kors, J. A., \& Van Herpen, G. (2005). The U wave in the electrocardiogram: a solution for a 100-yearold riddle. Cardiovasc Res., 67(2), 256-262. https://doi.org/10.1016/j.cardiores.2005.04.010

Heikes, R. P., \& Randall, D. A.(1995). Numerical integration of the shallow-water equations on a twisted icosahedral grid. part I: Basic design and results of tests. Mon. Wea. Rev., 123, 1862-1880. https://doi.org/10.1175/1520-0493(1995)123;1862:NIOTSW i2.0.CO;2

Heikes, R. P., \& Randall, D. A.(1995). Numerical integration of the shallow-water equations on a twisted icosahedral grid. part II: A detailed description of the grid and an analysis of numerical accuracy. Mon. Wea. Rev., 123, 1881-1887. https://doi.org/10.1175/1520-0493(1995)123;1881:NIOTSW ¿2.0.CO;2

Heikes, R. P., Randall, D. A., \& Konor, C. S. (2013). Optimized Icosahedral Grids: Performance of Finite-Difference Operators and Multigrid Solver. Mon. Wea. Rev., 141, 4450-4469. https://doi.org/10.1175/MWR-D-12-00236.1

Karaa, S., \& Zhang, J. (2003). Analysis of stationary iterative methods for the discrete convection-diffusion equation with a 9-point compact scheme. J. Comp. Appli Math., 154, 447-476. https://doi.org/10.1016/S0377-0427(02)00863-4

Kim, J. G., \& Huh, K. Y. (2000) Prediction of Transient Slab Temperature Distribution in the Re-heating Furnace of a Walking-beam Type for Rolling of Steel Slabs. ISIJ International, 40(11), 1115-1123. https://doi.org/10.2355/isijinternational.40.1115

Lee, D. (2015). Complete solution to seven-point schemes of discrete anisotropic Laplacian on regular hexagons. Applicable Analysis and Discrete Mathematics, 9, 180-197. https://doi.org/10.2298/AADM150310010L

Lee, D. (August, 2017). Analysis of Hexagonal Grid Finite Difference Methods for Anisotropic Laplacian Related Equations. Numer. Math. Theor. Meth. Appl., 10(3), August, 2017 (in print).

Lee, D., Tien, H. C., Luo, C. P. \& Luk, H-N. (2014). Hexagonal grid methods with applications to partial differential equations. International Journal of Computer Mathematics, 91(9), 1986-2009. https://doi.org/10.1080/00207160.2013.864392

Makarov, V. L., Mararov, S. V., \& Moskal'kov, M. N. (1993). Spectral properties of the difference Laplacian on a hexagonal mesh and their application. Differ. Equ., 29(7), 959-1118. 
McCartin, B. J. (2003). Eigenstructure of the equilateral triangle,part I: the Dirichlet problem. SIAM Review, 45(2), 267-287. https://doi.org/10.1137/S003614450238720

McCartin, B. J. (2002). Eigenstructure of the equilateral triangle,part II: the Neumann problem. Mathematical Problems in Engineering, 8(5), 517-539. https://doi.org/10.1080/1024123021000053664

Nickovic, S., Gavrilov, M. B., \& Tosic, I. A. (2002) Geostrophic Adjustment on Hexagonal Grids. Mon. Wea. Rev., 130, 668-683. https://doi.org/10.1175/1520-0493(2002)130;0668:GAOHG;2.0.CO;2

Pal, S., Lan, C., ..., \& Ma, Y. (2015). Symmetry boundary condition in dissipative particle dynamics. Journal of Computational Physics, V. 292, 287-299. https://doi.org/10.1016/j.jcp.2015.03.025

Pickering, W. M. (1986). On the solution of Poisson's equation in a regular hexagonal grid Using FFT Methods. JCP, 64, 320-333. https://doi.org/10.1016/0021-9991(86)90036-7

Strauss, W. A. (2008). Partial Differential Equation,An Introduction,2nd ed.. John Wiley \& Sons, Inc., Hoboken, NJ.

Suli,E. (1993). Convergence of Finite Volume Schemes for Poisson s Equation on Nonuniform Meshes. SIAM J. Numer. Anal., 28(5), 1419-1430. https://doi.org/10.1137/0728073

Xu, C. M., \& Soares, C. G. (2013). Comparisons of calculations with experiments on the ultimate strength of wide stiffened panels. Marine structures, 31, 82-101. https://doi.org/10.1016/j.marstruc.2013.01.003

Zhou, G., \& Fulton, S. R.(2009). Fourier Analysis of Multigrid Methods on Hexagonal Grids. SIAM J. Sci. Comput., 31(2), 1518-1538. https://doi.org/10.1137/070709566

\section{Copyrights}

Copyright for this article is retained by the author(s), with first publication rights granted to the journal.

This is an open-access article distributed under the terms and conditions of the Creative Commons Attribution license (http://creativecommons.org/licenses/by/4.0/). 